\title{
Determination of a temporally and spatially resolved Supernova rate from OB-stars within 5 kpc
}

\author{
J.G. Schmidt ${ }^{\star}$, M.M. Hohle, and R. Neuhäuser \\ Astrophysikalisches Institut und Universitäts-Sternwarte Jena, Schillergässchen 2-3, D-07745 Jena, Germany
}

Received 30 May 2005, accepted 11 Nov 2005

Published online later

Key words stars: evolution - stars: fundamental parameters - stars: statistics - binaries: general - supernovae: general

We spatially and temporally resolve the future Supernova (SN) rate in the Solar vicinity and the whole Galaxy by comparing observational parameters of massive stars with theoretical models for estimating age and mass and, hence, the remaining life-time until the SN explosion. Our SN rate derived in time and space for the future (few Myr) should be the same as in the last few Myr by assuming a constant rate.

From BVRIJHK photometry, parallax, spectral type, and luminosity class we compile a Hertzsprung-Russell diagram (H-R D) for 25027 massive stars and derive extinction, and luminosity, then mass, age, and remaining life-time from evolutionary models.

Within 600 pc our sample of SN progenitors and, hence, SN prediction, is complete, and all future SN events of our sample stars take place in $8 \%$ of the area of the sky, whereas $90 \%$ of the events take place in $7 \%$ of the area of the sky. The current $\mathrm{SN}$ rate within $600 \mathrm{pc}$ is increased by a factor of 5-6 compared with the Galactic rate. For a distance of $5 \mathrm{kpc}$ our sample is incomplete, nevertheless $90 \%$ of those SN events take place in only $12 \%$ of the area of the projected sky. If the SN rate in the near future is the same as the recent past, there should be unknown young neutron stars concentrated in those areas. Our distribution can be used as input for constraints of gravitational waves detection and for neutron star searches.

(C) 2006 WILEY-VCH Verlag GmbH \& Co. KGaA, Weinheim

\section{Introduction}

The search for gravitational wave (GW) events and pulsars using Einstein@home 1 can work more efficient with spatial limits. The spatially and temporally resolved future Supernova $(\mathrm{SN})$ rate is assumed to be roughly constant over some Myrs, and hence can indicate promising regions for the detection of probable GW sources such as precessing neutron stars (NSs) or binary NSs. The probability to find young NSs, which might be precessing and radiating GWs due to ellipticity, is larger in areas with a local increased SN rate. These sources can be detected with GW detectors (such as adLIGO). All sky surveys like Aasi et al. (2013) could be reduced to smaller areas in the sky by using our results. Thus the sensitivity of GW searches could be increased significantly.

100 to 140 NSs younger than $4 \mathrm{Myr}$ should be expected within $600 \mathrm{pc}$ from population synthesis (Popovet al. 2005), but only 15 of them are known yet in the ATNF pulsar catalogue (Manchester et al. 2005). The Galactic core collapse SN (ccSN) rate is estimated to $2.5_{-0.4}^{+0.8}$ events per century by counting SNe from Milky Way like galaxies (Tammann et al. 1994). The amount of ${ }^{26} \mathrm{Al}$ (Diehl et al. 2006) implies a rate of $(1.9 \pm 1.1) \mathrm{ccSNe}$ per century in our Galaxy. Grenier (2004) found 20 to $27 \mathrm{SNe}$ per Myr for the Gould Belt (Gould 1879), a local star form-

\footnotetext{
^ Corresponding author: e-mail: Schmidt.Janos@uni-jena.de

1 see http://einstein.phys.uwm.edu
}

ing region within $600 \mathrm{pc}$ (for an historical overview see Stothers \& Frogel 1974), by studying observed star counts. This local current rate is 5 to 6 times higher than the Galactic average. A spatially and temporally resolved SN rate of $21 \pm 5 \mathrm{SNe}$ per Myr within $600 \mathrm{pc}$ was published by Hohle, Neuhäuser \& Schutz (2010). They analysed the SN rate on the base of massive stars observed with both HIPPARCOS and 2MASS. Hohle et al. (2010) derive mass and age for $\mathrm{SN}$ progenitors by using different evolutionary models, and determine the remaining life-time. Their sample is complete for stars brighter than the limiting magnitude of $V=8.0 \mathrm{mag}$ (van Leeuwen 2007) for HIPPARCOS. Thus all B4V and earlier stars within a distance of $600 \mathrm{pc}$ are included, assuming a mean interstellar extinction of $a_{V}=1 \mathrm{mag} / \mathrm{kpc}$ for the solar vicinity (Lynga 1982).

In this work we enhance the analysis of the $\mathrm{SN}$ rate for more distant stars. We use additional and more recent databases to find more ccSN progenitors in the solar vicinity up to $5 \mathrm{kpc}$. Furthermore, while all spectral types in Hohle et al. (2010) are taken from the HIPPARCOS catalogue (Perryman et al. 1997) or SIMBAD, we also use more recent spectral classifications. Each step of the analysis is reconsidered and improved. Wolf-Rayet stars (WR) were not included in Hohle et al. (2010) but will be included in this work. For a better evaluation of the numbers we calculate the completeness of our sample to specify the areas in the sky which should be explored in more detail in the future. 
Table 1 Selection criteria for the luminosity classes and the spectral types.

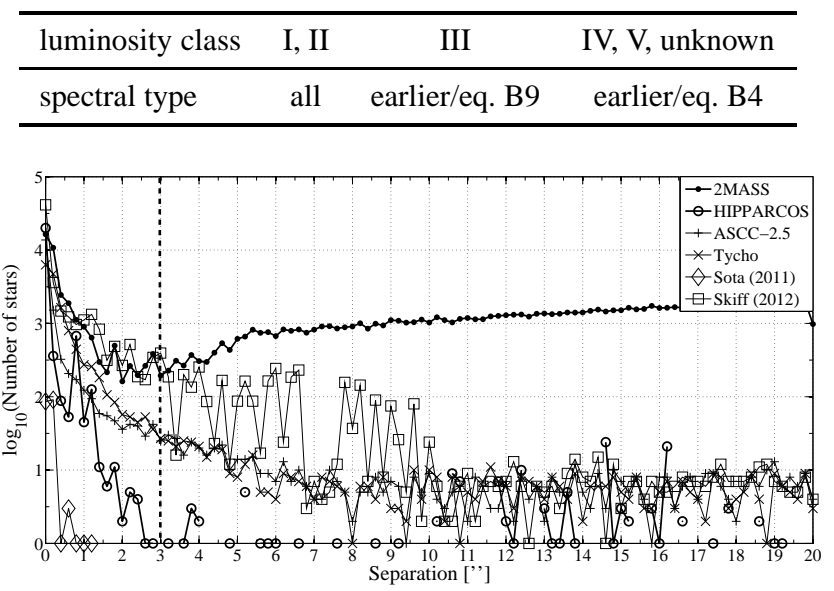

Fig. 1 Calculating the optimal aperture from the distribution of the separation of catalogue stars towards a sample star. The catalogues are $2 \mathrm{MASS}$, the new reduction of HIPPARCOS, ASCC-2.5, Tycho, the massive star survey by Sota et al. (2011) and the catalogue from Skiff (2013). The optimal aperture is the first local minimum and is found to be 3 .' 0 for HIPPARCOS and 2MASS, indicated by the dashed vertical line.

\section{The sample}

The base of the whole analysis is a set of potential SN progenitors $\left(M>8 M_{\odot}\right)$ that is queried for observational parameters. We select all ccSN progenitors regarding the spectral type classification (SpT): we cover all SpT for luminosity classes (LCs) I and II, all stars earlier than or equal to SpT B9 for LC III, and all known stars earlier than or equal to spectral type B4 with LC IV, V, or an unknown LC will be also included (Table 1). The basic set of stars comes from a select by criteria query in SIMBAD (see Appendix A), consisting of 18146 objects (as of 2013-03-04). We expand the sample from SIMBAD with the catalogues in Table 2 using the VizieR Online Database (Ochsenbein et al. 2000). There are 13741 additional stars fulfilling our selection criteria from Table 1 plus 226 WR stars from van der Hucht (2001). Thus our sample expands to 32113 stars. Some catalogues are including bright stars not belonging to our Galaxy. We avoid an extragalactic overpopulation in our sample by excluding 7165 stars in the sky area of the Large and the Small Magellanic Cloud.

We use a cross correlation to re-identify stars in different catalogues. To avoid a misidentification of stars, we determine an optimal aperture from counting the spatial separation of the catalogue stars towards a sample star within a maximum aperture radius of $20^{\prime \prime}$ (Figure 1). Given the location of the minimum in the separation distribution we consider an optimal aperture of 3 .'0 for 2MASS and HIPPARCOS coordinates and use this result for all other catalogues. The star with the minimal spatial separation between sam-
Table 2 Number of identified stars in different catalogues within a search aperture of $3 . \prime 0$, the references are [1] Skrutskie et al. (2006), [2] Kharchenko \& Roeser (2009), [3] Skiff (2013), [4] Perryman et al. (1997), [5] van Leeuwen (2007), [6] Zacharias et al. (2005), [7] Schroeder et al. (2004), [8] Hoeg et al. (1997), [9] Zacharias et al. (2013), [10] Bondarenko \& Perevozkina (1996), [11] Brancewicz \& Dworak (1980), [12] Docobo \& Andrade (2006), [13] Perevozkina \& Svechnikov (1999), [14] Pourbaix et al. (2004), [15] Surkova \& Svechnikov (2004), [16] van der Hucht (2001), [17] Sota et al. (2011), [18] Dommanget \& Nys (2002), [19] Mason et al. (2010)

\begin{tabular}{lll}
\hline catalogue name & stars & ref. \\
\hline 2MASS & 22755 & {$[1]$} \\
Allsky Comp. Cat. of 2.5 mio stars & 19597 & {$[2]$} \\
Cat. of Stellar Spectral Class. & 9087 & {$[3]$} \\
HIPPARCOS Main Cat. & 6613 & {$[4]$} \\
HIPPARCOS, New Reduction & 6613 & {$[5]$} \\
NOMAD & 23193 & {$[6]$} \\
Hipparcos parallaxes of O stars & 154 & {$[7]$} \\
TYCHO Main Part & 18415 & {$[8]$} \\
UCAC4 & 22387 & {$[9]$} \\
early-type contact binaries & 31 & {$[10]$} \\
Parameters of eclipsing binaries & 163 & {$[11]$} \\
Spec. subcomp. in mult. systems & 4 & {$[12]$} \\
Cat. of eclipsing binaries parameters & 9 & {$[13]$} \\
$9^{\text {th }}$ Cat. of Spec. Binary Orbits & 531 & {$[14]$} \\
Semi-detached eclipsing binaries & 37 & {$[15]$} \\
$7^{\text {th }}$ Cat. of Galactic WR stars & 226 & {$[16]$} \\
Galactic O-Star Spectral Survey & 184 & {$[17]$} \\
CCDM & 2506 & {$[18]$} \\
WDS & 2595 & {$[19]$} \\
\hline total number of different stars & 24948 & \\
\hline
\end{tabular}

ple and catalogue coordinates within the optimal aperture is identified to be the correct counterpart. If identifiers like DM, HD or HIP number are available, we check those as well. The numbers of identified sample stars in different catalogues are listed in Table 2

The spatial distribution in galactic coordinates, of our whole sample stars is plotted in Figure 2.

\section{Stellar input parameters}

We collect for each star observational parameters i.e. the spectral type, BVRIJHK photometry, and the distance from the catalogues mentioned above (see Table 3 for detailed list).

\subsection{Temperature}

The effective temperature is calculated from the most recently published SpT available in the literature by using 
Table 3 Stellar input parameters for ten progenitor stars of our sample, sorted by the ascending relative error of the parallaxes. The complete table with 24948 stars plus 79 resolved massive companions in multiples subsection 3.3) will be available at the CDS/ViZieR online data base. See Section $2 \& 3$ for references.

\begin{tabular}{|c|c|c|c|c|c|c|c|c|c|}
\hline$\#$ & ID & $\begin{array}{c}\text { B } \\
{[\mathrm{mas}]}\end{array}$ & $\begin{array}{c}\mathrm{V} \\
{[\mathrm{mas}]}\end{array}$ & $\begin{array}{c}\mathbf{J} \\
{[\mathrm{mas}]}\end{array}$ & $\begin{array}{c}\mathrm{H} \\
{[\mathrm{mas}]}\end{array}$ & $\begin{array}{c}\mathrm{K} \\
{[\mathrm{mas}]}\end{array}$ & $\begin{array}{c}\pi \\
{[\mathrm{mas}]}\end{array}$ & SpType & $\begin{array}{c}T_{\text {eff }} \\
{[\mathrm{K}]}\end{array}$ \\
\hline 1 & $\alpha$ Mus & $513(2)$ & $2.681(2)$ & $3.18 \pm 0.25$ & $3.17 \pm 0.21$ & $3.25 \pm 0.28$ & $10.44 \pm 0.11$ & B2IV-V & $22000_{-3400}^{+3300}$ \\
\hline 2 & $\eta$ Cen & $2.174(3)$ & $2.328(4)$ & $2.75 \pm 0.29$ & $2.78 \pm 0.21$ & $2.75 \pm 0.26$ & $10.78 \pm 0.21$ & $\mathrm{~B} 2 \mathrm{Ve}$ & $22000_{-3400}^{+3300}$ \\
\hline 3 & $\beta$ Lup & $2.493(2)$ & $2.668(2)$ & $3.15 \pm 0.21$ & $3.16 \pm 0.17$ & $3.25 \pm 0.27$ & $8.61 \pm 0.18$ & B2IV & $21150_{-3550}^{+3250}$ \\
\hline 4 & HR 4898 & $3.834(2)$ & $3.993(2)$ & $4.56 \pm 0.23$ & $4.56 \pm 0.08$ & $4.53 \pm 0.02$ & $7.95 \pm 0.17$ & B1V & $25400_{-4600}^{+3400}$ \\
\hline 5 & $\zeta \mathrm{Oph}$ & $2.598(3)$ & $2.569(3)$ & $2.53 \pm 0.30$ & $2.67 \pm 0.21$ & $2.68 \pm 0.27$ & $9.00 \pm 0.20$ & O9.5IV & $27100_{-8150}^{+2} 400$ \\
\hline 6 & $\alpha \operatorname{Lup}$ & $2.131(2)$ & $2.282(2)$ & $2.63 \pm 0.26$ & $2.72 \pm 0.20$ & $2.67 \pm 0.24$ & $7.09 \pm 0.17$ & B2IV & $21150_{-3550}^{+3250}$ \\
\hline 7 & HR 5193 & $3.280(3)$ & $3.460(3)$ & $3.71 \pm 0.21$ & $3.74 \pm 0.20$ & $4.01 \pm 0.04$ & $6.51=$ & B2Vnep & $22000_{-3400}^{+3300}$ \\
\hline 8 & $\phi$ Cen & $3.618(3)$ & $3.811(2)$ & $4.63 \pm 0.28$ & $4.46 \pm 0.26$ & $4.49 \pm 0.02$ & $6.27 \pm 0.17$ & $\mathrm{~B} 2 \mathrm{~V}$ & $22000_{-3400}^{+3300}$ \\
\hline 9 & $\zeta \mathrm{Cas}$ & $3.490(2)$ & $3.671(2)$ & $4.14 \pm 0.30$ & $4.25 \pm 0.25$ & $4.25 \pm 0.04$ & $5.56 \pm 0.16$ & B2IV & $21150_{-3550}^{+3250}$ \\
\hline 10 & $v$ Sco & $2.502(4)$ & $2.674(4)$ & $3.05 \pm 0.23$ & $3.11 \pm 0.19$ & $3.18 \pm 0.26$ & $5.72 \pm 0.18$ & B2IV & $21150_{-3550}^{+3250}$ \\
\hline
\end{tabular}

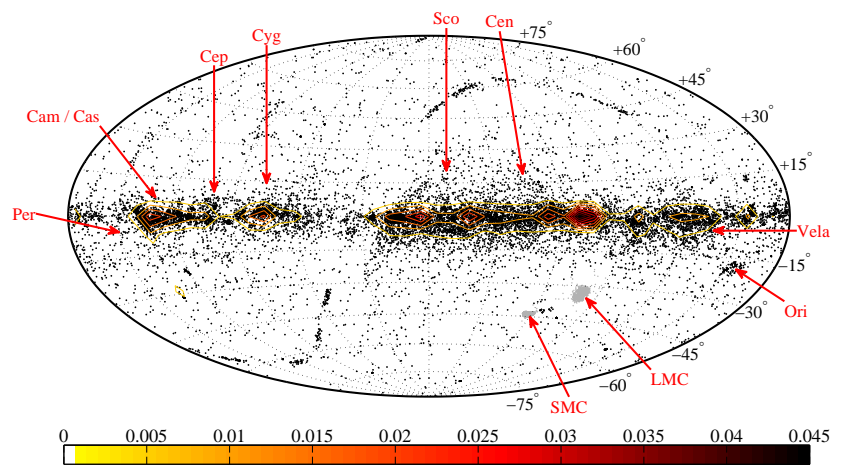

Fig. 2 The spatial distribution of our sample stars in galactic coordinates created with SIMBAD and various VizieR catalogues (see section 2), the grey dots indicate stars in the sky area of the Large and Small Magellanic Cloud which we exclude from our sample.

data from Lang (1992) and for main sequence stars from Kenyon \& Hartmann (1995). The temperature error is calculated from the given uncertainty in the SpT. If there is no given uncertainty we assume an error of the SpT by \pm 1 subclass. We interpolate the temperature between neighbouring grid points for a decimal SpT linearly. For spectral types without a LC (3886 stars) we conservatively assume dwarf stars. If there is an uncertain LC (3800 stars), we consider the class with the lower luminosity (e.g. II/III is set to III). In total we collect full spectral classification for 24120 stars.

\subsection{Photometry and Extinction}

We use BVRIJHK photometry with Johnson $B V$ from the HIPPARCOS and Tycho catalogues given in Kharchenko \& Roeser (2009), $B V$ from UCAC4 Zacharias et al. (2013), the Cousins JHK photometry from 2MASS, and $B V R I J H K$ from SIMBAD. Having two different fluxes $X$ and $V$ the interstellar reddening $A_{V}$ due

2 taken from Schmidt-Kaler (1982) to interstellar extinction is calculated with the measured colour $(X-V)_{m}$ and the modelled colour $(X-V)_{0}$ according to:

$$
A_{V}=\frac{(X-V)_{m}-(X-V)_{0}}{\frac{A_{X}}{A_{V}}-1}
$$

We compute $A_{V}$ for the colours (B-V), (V-J), (V-H), (V$\mathrm{K}),(\mathrm{V}-\mathrm{R}),(\mathrm{B}-\mathrm{I}),(\mathrm{B}-\mathrm{R}),(\mathrm{R}-\mathrm{I}),(\mathrm{J}-\mathrm{H}),(\mathrm{J}-\mathrm{K})$, and (V-I) with model colours from Bessell et al. (1998). The model depends on $\log g$ and temperature, and hence on spectral classification. As an example Figure 3 shows the $A_{V}$ calculated from $(\mathrm{J}-\mathrm{K})$ and $(\mathrm{B}-\mathrm{V})$ as well as the number density of the values to emphasize the most frequent data points. The results of the error weighted linear fits are listed in Table 4 The final $A_{V}$ is calculated as an error weighted mean using all the values from the different colours mentioned above. Since the constants $\frac{A_{X}}{A_{Y}}$ published by Cardelli et al. (1989), Rieke \& Lebofsky (1985) and Savage \& Mathis (1979) are slightly different, the mean of these values is used.

WR stars have typically very broad emission lines, so we use available Smith narrowband photometry and extinction $A_{v}$ given by van der Hucht (2001) and calculate $A_{V}=0.9149 \cdot A_{v}$ as recommended in their paper.

We distinguish between an excess due to circumstellar dust and interstellar reddening by using infrared colourcolour diagrams (e.g. Figure 4p. There are 679 stars with an infrared excess due to circumstellar dust of more than $3 \sigma$ (colour error) in the JHK colour-colour diagram, which we take into account and correct for. Thus we avoid nonphysical values of $A_{V}$.

\subsection{Multiplicity}

Unresolved multiple stars would appear more luminous than resolved stars, hence the measured flux of unresolved multiple systems needs to be distributed among the components. Spectroscopic binaries are identified with catalogues from Brancewicz \& Dworak (1980), Bondarenko \& Perevozkina (1996), Pourbaix et al. (2004), Perevozkina \& Svechnikov 


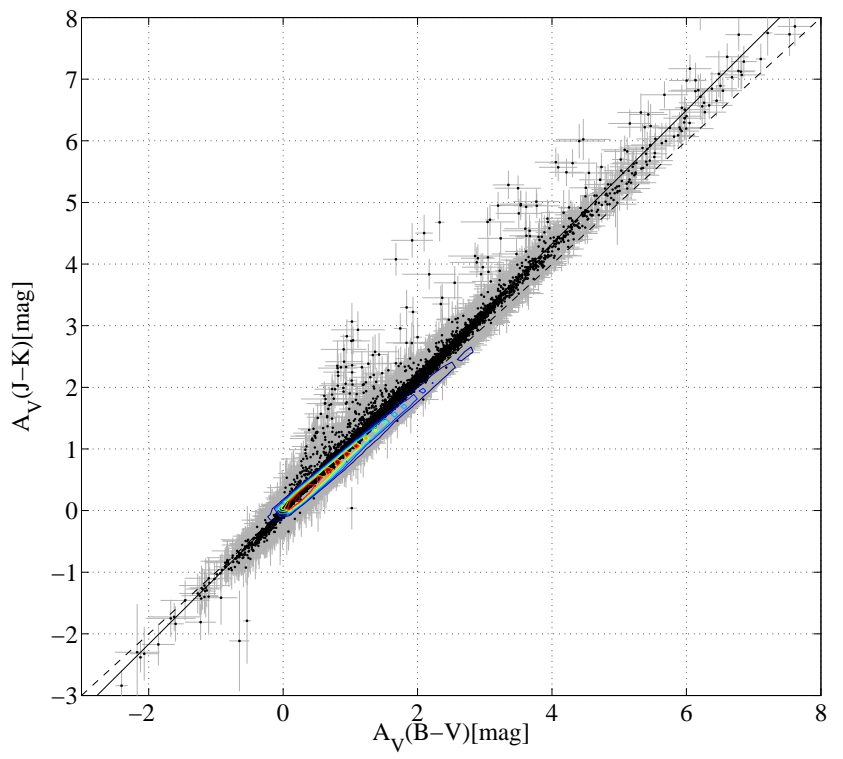

Fig. 3 Comparison of the derived $A_{V}$ using the colours $\mathrm{B}-\mathrm{V}$ and $\mathrm{J}-\mathrm{K}$ with $1 \sigma$ error bars. The one to one relation is indicated with a dashed line, the black line indicates the error weighted linear fit, and the coloured region shows the number density of stars from low (blue) to high (red). See Table 4 for the results of the other colour combinations.

(1999), Dommanget \& Nys (2002), Mason et al. (2010), Docobo \& Andrade (2006), Surkova \& Svechnikov (2004) and the SIMBAD object type (Table 5). We assume an identical interstellar extinction for unresolved members of a particular multiple and correct the apparent measured magnitudes for the components with:

$$
\begin{aligned}
X_{n}= & V_{n, m}-V_{s y s, m}-(X-V)_{s y s, 0} \\
& +(X-V)_{n, 0}+X_{s y s, m}
\end{aligned}
$$

$X_{s y s, m}$ indicates the measured magnitude of the system in the band $\mathrm{X}$ and $V_{n, 0}$ is the magnitude in the V-band of the $n$ th component. Thus we take into account the spectral classification and the measured magnitudes of the resolved components.

In our whole sample there are 4332 stars (17\% of our whole set) known to be members of 2131 multiple systems. This number is too small given the fact that $60-80 \%$ of the massive stars are expected to be components of a multiple system (Zinnecker \& Yorke 2007). For the visually resolved multiple systems we list all resolved components with spectral classifications fulfilling our criteria from Table 1 as additional entries in Table 3 .

In total we count 24948 stars in Table 3 plus 79 additional visual resolved massive companions. There are 925 unresolved multiples with additional known components not fulfilling our selection criteria, and 1197 unresolved multiples where nothing else (except their existence) is known about the companions, which we assume to be binaries with the same spectral type.
Table 4 The results of the error weighted fits $A_{V 1}=$ $m \cdot A_{V 2}+n$, where $A_{V 1}$ and $A_{V 2}$ are interstellar extinction values derived from the named colours, $\bar{\sigma}_{A_{V 1,2}}$ is the median of the respective error in mag, \# is the number of stars with an error smaller than $3 \bar{\sigma}_{A_{V 1,2}}$ that are used for the fit. All results are consistent with a one-to-one relation and an offset of $n=0$.

\begin{tabular}{cccccc}
\hline$A_{V 1}$ & $A_{V 2}$ & $3 \bar{\sigma}_{A_{V 1}}$ & $3 \bar{\sigma}_{A_{V 2}}$ & $\#$ & $m$ \\
\hline B-V & V-J & 0.35 & 0.22 & 10399 & $0.7 \pm 1.8$ \\
B-V & V-H & 0.35 & 0.23 & 10570 & $0.9 \pm 1.8$ \\
B-V & V-K & 0.35 & 0.17 & 10618 & $1.0 \pm 2.7$ \\
B-V & J-H & 0.35 & 1.63 & 10296 & $1.1 \pm 0.3$ \\
B-V & J-K & 0.35 & 0.85 & 10315 & $1.1 \pm 0.4$ \\
V-J & V-H & 0.22 & 0.23 & 11973 & $1.2 \pm 1.6$ \\
V-J & V-K & 0.22 & 0.17 & 11921 & $1.3 \pm 2.0$ \\
V-J & J-H & 0.22 & 1.63 & 11965 & $1.5 \pm 1.5$ \\
V-J & J-K & 0.22 & 0.85 & 11987 & $1.5 \pm 1.5$ \\
V-H & V-K & 0.23 & 0.17 & 12057 & $1.1 \pm 1.6$ \\
V-H & J-H & 0.23 & 1.63 & 12131 & $1.2 \pm 0.9$ \\
V-H & J-K & 0.23 & 0.85 & 12097 & $1.2 \pm 1.0$ \\
V-K & J-H & 0.17 & 1.63 & 11865 & $1.1 \pm 1.2$ \\
V-K & J-K & 0.17 & 0.85 & 11923 & $1.1 \pm 1.2$ \\
\hline
\end{tabular}

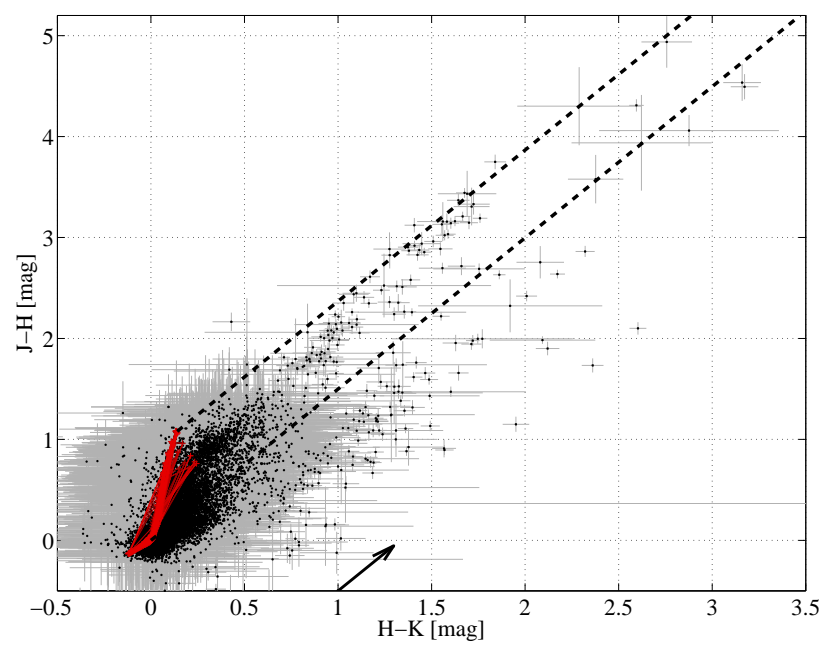

Fig. 4 The colour-colour diagram shows the measured (H-K) vs. (J-H) for our set of stars (black dots). The red lines are showing the modelled colours by Bessell et al. (1998) from dwarfs to hyper giants, the black arrow indicates interstellar extinction of $5 \mathrm{mag}$, and the upper and lower dashed lines are indicating the reddening of stars with an effective temperature of 3500 and $50000 \mathrm{~K}$. Stars to the lower-right of the lower dashed line have circumstellar material.

\subsection{Distance}

The distance is calculated from the trigonometric parallax $\pi$ using the HIPPARCOS data of the recent reduction by van Leeuwen (2007). If there is no HIPPARCOS value available for a star, the Tycho data or the catalogue from Kharchenko \& Roeser (2009) is used. The trigonometric parallax is available for 18300 sample stars. Among them, we count 11862 stars with $\pi \geq 0.2$ mas, 10102 stars with 
Table 5 Numbers of multiple systems found in our sample with different catalogues

\begin{tabular}{ll}
\hline Catalogue & multiple systems \\
\hline CCDM & 1913 \\
WDS & 2844 \\
Bondarenko \& Perevozkina (1996) & 33 \\
Brancewicz \& Dworak (1980) & 175 \\
Docobo \& Andrade (2006) & 5 \\
Perevozkina \& Svechnikov (1999) & 9 \\
Pourbaix et al. (2004) & 574 \\
Surkova \& Svechnikov (2004) & 39 \\
\hline
\end{tabular}

Table 6 Number of stars with measured parallax $\pi$ and transformed parallax $\pi_{t}$ higher than its error $\sigma$.

\begin{tabular}{lcccc}
\hline$\left|\frac{\pi}{\sigma_{\pi}}\right|$ & $\geq 5$ & $\geq 3$ & $\geq 2$ & $\geq 1$ \\
\hline measured $\pi$ & 1913 & 3281 & 5293 & 10102 \\
transformed $\pi_{t}$ & 1954 & 3761 & 13883 & 18047 \\
both $\pi$ and $\pi_{t}$ & 1912 & 3276 & 5264 & 9978 \\
\hline
\end{tabular}

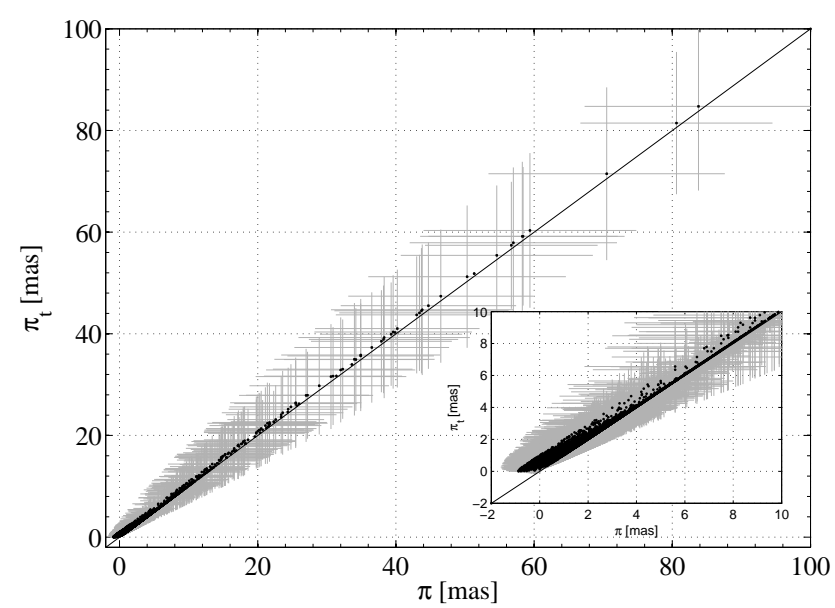

Fig. 5 Transformed parallax $\pi_{t}$ compared to the measured parallax $\pi$ using the transformation from Smith \& Eichhorn (1996) for 6407 stars with $\left|\pi_{t}-\pi\right| \leq 1$ mas. The solid line indicates the one-to-one relation. The interval up to 10 mas is shown in the lower right.

a value higher than its error $\left|\frac{\sigma_{\pi}}{\pi}\right| \leq 1$, and 6191 with a negative parallax. All parallaxes $\pi$ are transformed to the expectation value $\pi_{t}$ using Eq. 21 from Smith \& Eichhorn (1996). The influence of this transformation on parallaxes with large errors is seen in Table 6 . There would be an overpopulation of 7945 stars with significantly underestimated distances resulting in inconsistent luminosities compared to their SpT and LC.

Since negative parallaxes are converted to small positive values within their errorbars, we flag those values as uncertain to exclude them from further calculations. We use the transformation to utilize those parallaxes which are comparable to their expectation value and select 6407 stars with $\left|\pi_{t}-\pi\right| \leq 1$ mas (Figure 5). Thus we avoid uncertain par-

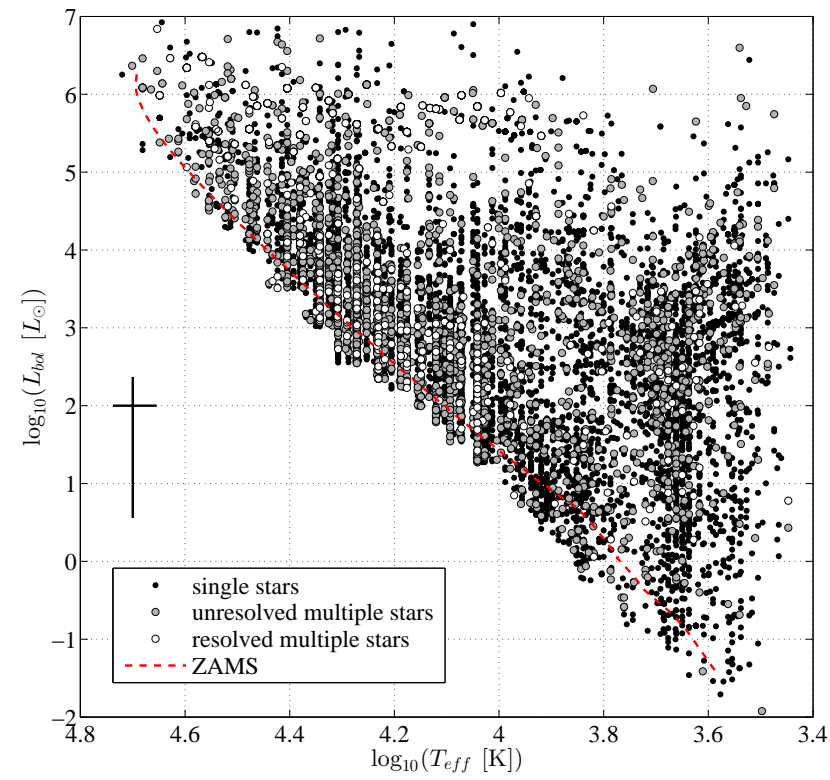

Fig. 6 Our derived H-R D: the red dashed line marks the zero age main sequence (ZAMS), grey dots indicate resolved stars in a multiple system, black circles denote unresolved multiples, and black dots stand for single stars, the mean errorbar is indicated by the black cross on the left side.

allaxes with large errors but include measurements accurate down to sub-mas.

The results of the remaining part of the paper are calculated with and without an applied transformation to study systematic uncertainties that may arise from Eq. 21 by Smith \& Eichhorn (1996). All values are consistent within their errorbars (see Appendix B.

\subsection{Luminosity}

The bolometric luminosity in solar units is

$$
L_{b o l}=10^{0.4\left(5 \log _{10} d-5+4.74 m a g-B C_{V}-m_{V}+A_{V}\right)}
$$

with the distance $d$ in pc, the bolometric correction in the Vband $B C_{V}$, the apparent magnitude in the V-band $m_{V}$, and the interstellar extinction in the V-band $A_{V}$. The $B C_{V}$ from Bessell et al. (1998) is calibrated to $M_{b o l} \odot=4.74 \mathrm{mag}$ and is consistent with the apparent magnitude of $V_{\odot}=$ $-26.76 \mathrm{mag}$ as mentioned in their paper. This test is suggested by Torres (2010), because the zero point of the $B C_{V}$ is arbitrary, but the bolometric magnitude of the sun is not.

\section{Derived stellar parameters: mass, age, and life-time}

We estimate mass and age by comparing our calculated bolometric luminosities and temperatures with the theoretical H-R D from Bertelli et al. (1994), Schaller et al. (1992), and Claret (2004). The differences of these models among each other are described in Hohle et al. (2010). Our mass 


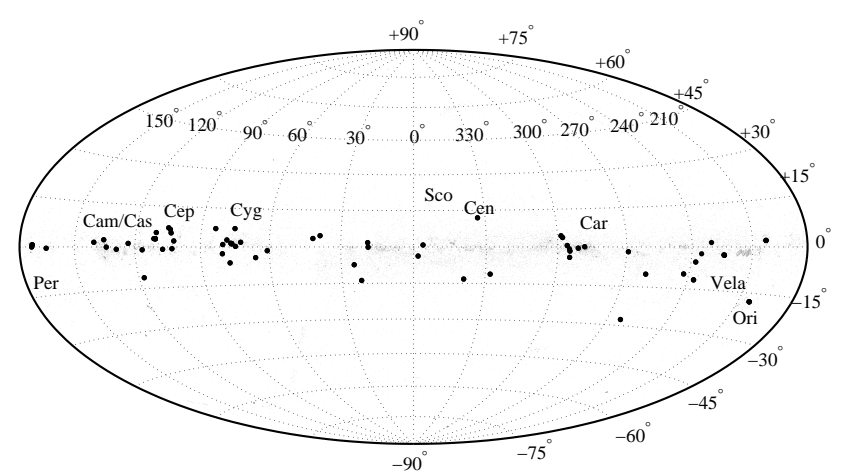

Fig. 7 The spatial distribution of 146 binaries, where both components have at least $8 M_{\odot}$ (black dots) and the entire sample (grey dots).

limit of $8 \mathrm{M}_{\odot}$ corresponds to a main sequence star with $\log \left(L_{b o l} / L_{\odot}\right) \geq 3.41$ and $\log \left(T_{e f f} /[K]\right) \geq 4.23$ regarding the model from Claret (2004). Mass and age are calculated from the error-weighted closest grid-points per model. We then calculate the mean mass and mean age from the different models. For stars with $M \geq 35 M_{\odot}$ we use the models from Claret (2004) and Schaller et al. (1992). The theoretical H-R D from Bertelli et al. (1994), Schaller et al. (1992), and Claret (2004) are applied for stars with $M<35 M_{\odot}$. For WR stars we only use the model from Meynet \& Maeder (2003).

There are 8143 stars below the zero-age-main-sequence (ZAMS) due to large uncertainties of the distance, which are conservatively assumed to have at least a luminosity consistent with the ZAMS (as seen in Figure 6. So far we cannot distinguish between stars with underestimated distances and misidentified subdwarfs that also lie below the ZAMS, thus we flag those stars. From our 6407 stars from the subsample with sufficiently measured distances subsection 3.4, we correct 2663 stars to be on the ZAMS. Those stars probably have erroneous distances which are not consistent to be within $1 \mathrm{kpc}$, however they should have distances smaller than $5 \mathrm{kpc}$ due to the completeness of the catalogues for the parallax and the apparent magnitudes.

Since the spectroscopic binary catalogues from Table 5 list dynamical masses, we use these instead of model dependent masses if available. For main sequence stars those dynamical masses are consistent with our masses estimated from the H-RD within their $1 \sigma$ errorbars (Docobo \& Andrade 2013).

Massive multiple systems where at least two components have masses above $8 M_{\odot}$ are important for the study of the evolution of compact binary systems. The spatial distribution of those massive binaries is shown in Figure 7 (listed in Table C1). In the near future, we will also study the binary evolution and interaction in those systems.

The empirical mass luminosity relation $L \sim M^{\beta}$ yields $\beta=3.93 \pm 0.78$ for main sequence stars from our sample and is plotted in Figure 8. That error weighted result is consistent with the values from Hilditch (2001) with $\beta=4.0$

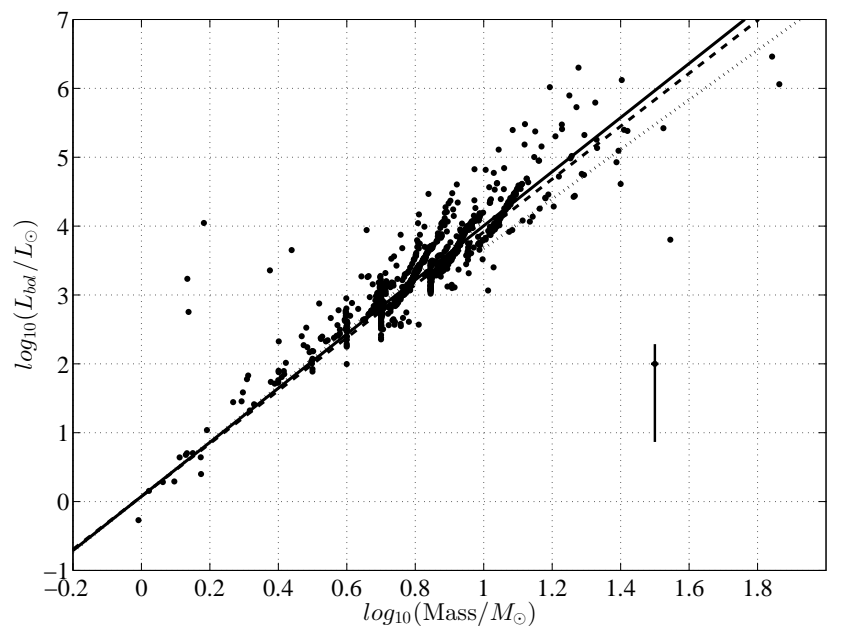

Fig. 8 Derived error weighted mass luminosity relation $L \sim M^{\beta}$ with $\beta=3.93 \pm 0.78$ (solid line) for main sequence stars compared to published values from Hilditch (2001) with $\beta=4.0$ for stars with $M \leq 10 M_{\odot}$ and $\beta=3.6$ for stars with a higher mass (dotted line), and $\beta=3.84$ from Andersen (1991) (dashed line). The black cross indicates the mean errorbar.

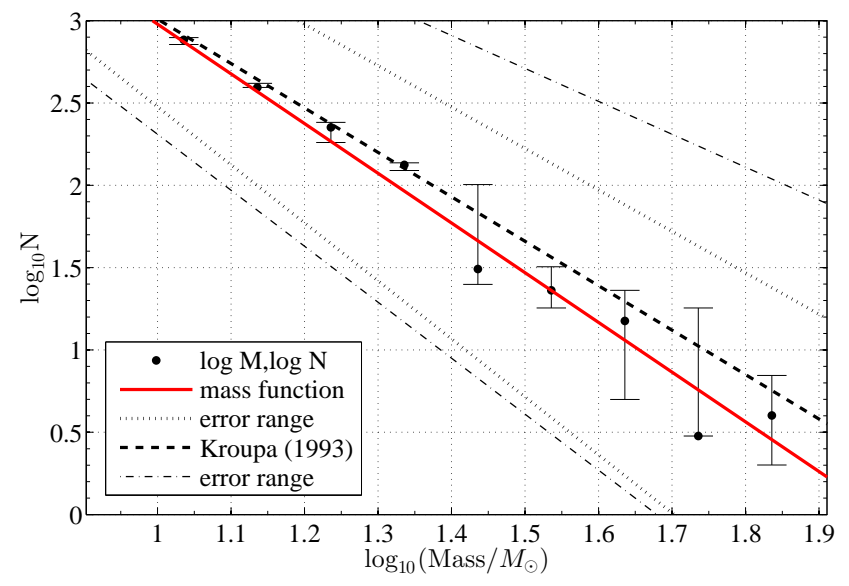

Fig.9 Our derived mass function $N \sim M^{\Gamma}$ (red solid line) with $\Gamma=-3.1 \pm 0.5$ for stars with $M \gtrsim 8 M_{\odot}$ compared to the initial mass function from Kroupa et al. (1993) with $\Gamma=-2.7 \pm 0.7$ (black dashed line). The errorbars of the individual masses (black dots) indicate the $1 \sigma$ confidence interval.

for stars with a mass less than $10 M_{\odot}$ and $\beta=3.6$ for stars with a higher mass, from Andersen (1991) with $\beta=3.84$, and with $\beta=3.66 \pm 0.12$ from Hohle et al. (2010).

As another consistency check we calculate the mass function $N \sim M^{\Gamma}$ for massive stars by using an error weighted fit yielding $\Gamma=-3.1 \pm 0.5$ (see Figure 9), that is in good agreement with $\Gamma=-2.7 \pm 0.7$ from the initial mass function by Kroupa et al. (1993).

The H- and He-burning phases specify the life-time of a star due to the long duration (Myr) while other burning processes like C-burning lasts a few kyr only. The life-time 
Table 7 List of $L_{b o l}$, mass, age, remaining life-time $\tau_{r}$, and number $\mathrm{N}$ of known additional components of the first ten SN progenitors in Table 3, with $1 \sigma$ errors. Uncertain remaining life-times are marked with a leading colon. The complete table with 25027 entries will be published on the CDS/ViZieR online data base.

\begin{tabular}{llrrrrr}
\hline$\#$ & name & $\begin{array}{r}L_{\text {bol }} \\
10^{3}\left[L_{\odot}\right]\end{array}$ & $\begin{array}{r}\text { mass } \\
{\left[M_{\odot}\right]}\end{array}$ & $\begin{array}{r}\text { age } \\
{[\mathrm{Myr}]}\end{array}$ & $\begin{array}{r}\tau_{r} \\
{[\mathrm{Myr}]}\end{array}$ & $\mathrm{N}$ \\
\hline 1 & $\alpha$ Mus & $5.3 \pm 2.4$ & $8.5(2)$ & $15 \pm 3$ & 14 & 4 \\
2 & $\eta$ Cen & $7.2 \pm 3.4$ & $8.7(2)$ & $20 \pm 3$ & 8 & 0 \\
3 & $\beta$ Lup & $6.7 \pm 3.1$ & $8.2(3)$ & $28 \pm 6$ & $: 12$ & 1 \\
4 & HR 4898 & $3.7 \pm 1.7$ & $8.9(1)$ & $3 \pm 1$ & 24 & 1 \\
5 & $\zeta$ Oph & $21.9 \pm 2.5$ & $12.5(1)$ & $13 \pm 1$ & 2 & 0 \\
6 & $\alpha$ Lup & $15.4 \pm 7.4$ & $9.6(2)$ & $23 \pm 1$ & $: 3$ & 2 \\
7 & HR 5193 & $6.7 \pm 3.3$ & $8.7(2)$ & $19 \pm 4$ & 9 & 2 \\
8 & $\phi$ Cen & $4.6 \pm 2.3$ & $8.3(2)$ & $14 \pm 2$ & 17 & 0 \\
9 & $\zeta$ Cas & $6.2 \pm 3.1$ & $8.1(2)$ & $28 \pm 5$ & 4 & 0 \\
10 & $v$ Sco & $16.1 \pm 8.0$ & $9.7(3)$ & $23 \pm 1$ & $: 7$ & 0 \\
\hline
\end{tabular}

of a star can be calculated from its mass by using models of Kodama (1997) and Maeder \& Meynet (1989). The remaining life-time $\tau_{r}$ of a star is the difference between the mean of the expected modelled life-time $\tau_{m}$ and the age $\tau_{\text {age }}$.

$$
\tau_{r}=\tau_{m}-\tau_{\text {age }}
$$

Since $\tau_{m}$ is not well calibrated for the theoretical H-R D, there are 9563 stars in our sample with a negative remaining life-time. All stars with a negative remaining life-time are randomly set to plausible life-times according to the distribution of positive life-times of their respective mass bin. Hence, the remaining life-time distribution of every mass bin itself is not changed by this correction. The corrected life-times are marked with a leading colon in Table 7 If there is no positive life-time distribution for a mass bin, we flag those life-times with a question mark. We count 2643 stars with a corrected remaining life-time from the subsample of 6407 stars with sufficient distances subsection 3.4.

The calculated $L_{b o l}$, the mass, the age, the remaining life-time $\tau_{r}$, and the number $\mathrm{N}$ of known additional components are listed in Table 7.

\subsection{Completeness}

The substantial completeness of our sample is conservatively calculated in Figure 10 by dividing the cumulative number of stars at a certain distance by the value from a fitted power-law growth $N \sim d^{\Phi}$. The scale height of the spiral arm population is $\approx 120 \mathrm{pc}$ and the young disc population has $\approx 200 \mathrm{pc}$ in Mihalas \& Binney (1981). Our sample corresponds to a cubic growth $(\Phi=3)$ for distances from 1 to $170 \mathrm{pc}$ as expected for increasing the observed spheric volume and counting the star numbers. Thus, the scale height of our stars is $170 \mathrm{pc}$. The scale height of the Galactic disc yields $260 \mathrm{pc}$ (Harding et al. 2001). The best fit is $\Phi=1.4$ from $170 \mathrm{pc}$ to $260 \mathrm{pc}$, which agrees to a less

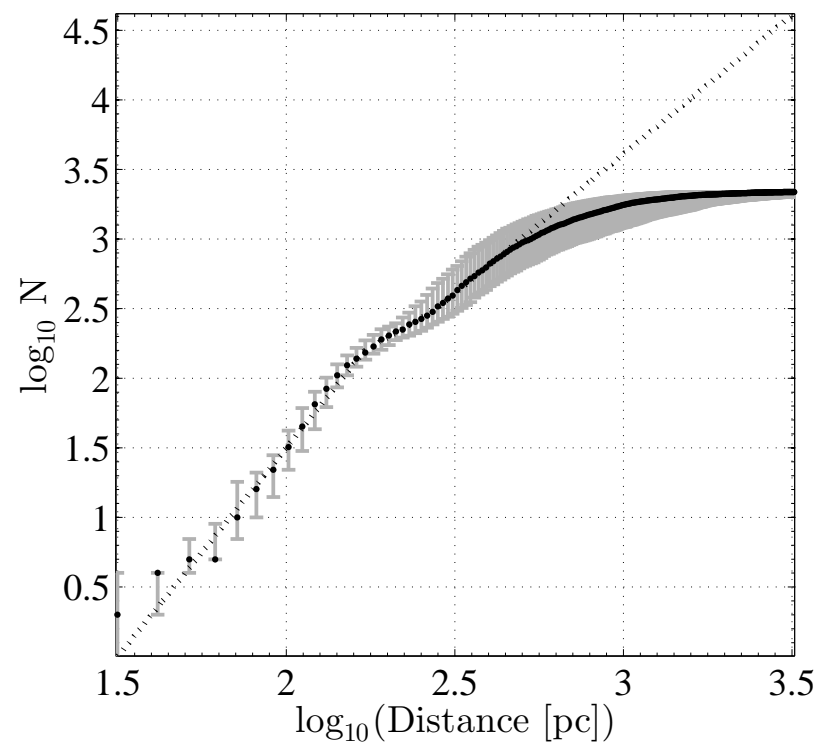

Fig. 10 The cumulative number of stars $N$ (black dots with grey errorbars) versus the distance $d$; our sample is consistent with a powerlaw (black dotted line) $N \sim d^{\Phi}$ of $\Phi=3.0$ for distances between 1 to $170 \mathrm{pc}, \Phi=1.4$ from $170 \mathrm{pc}$ to $260 \mathrm{pc}$, and $\Phi=2.0$ for $260 \mathrm{pc}$ to $500 \mathrm{pc}$. Thus we estimate the completeness of our sample with the powerlaw index of 2.0 compared to the cumulative number. For a distance of $500 \mathrm{pc}$ our sample is complete to $97 \%, 85 \%$ for $600 \mathrm{pc}, 45 \%$ for $1 \mathrm{kpc}, 6 \%$ for $3 \mathrm{kpc}$, and $2 \%$ for $5 \mathrm{kpc}$. The grey errorbars are obtained by adding or subtracting $1 \sigma$ from the parallax.

dense populated region up to the scale height. Corresponding to an increase of the observed area within the Galactic disc a quadratic growth $(\Phi=2)$ fits for distances from $260 \mathrm{pc}$ to $500 \mathrm{pc}$. After $500 \mathrm{pc}$ the incompleteness of our sample increases, hence we cannot longer fit a powerlaw growth to the cumulative number. Dividing the cumulative number by the value from the continued quadratic powerlaw for distances higher than $500 \mathrm{pc}$ our set of stars is $85 \%$ complete within $600 \mathrm{pc}, 45 \%$ within $1 \mathrm{kpc}, 6 \%$ within $3 \mathrm{kpc}$, and $2 \%$ within $5 \mathrm{kpc}$. Thus, for distances larger than $1 \mathrm{kpc}$ our sample is highly incomplete.

\section{Discussion on Supernova progenitor distribution}

If the current local $\mathrm{SN}$ rate is roughly constant for some 10 to $20 \mathrm{Myr}$, then all ccSN progenitors with a mass higher than $8 M_{\odot}$ and a remaining life-time shorter than $10 \mathrm{Myr}$ are indicators for regions with young neutron stars and potential GW sources. We can discuss our observed set of stars only within the next $10 \mathrm{Myr}$ because afterwards new star formation must be taken into account e.g. by performing a population synthesis.

The regions with a locally increased $\mathrm{SN}$ rate within $600 \mathrm{pc}$ are shown of the whole sky with a binsize of $7^{\circ} \times 7^{\circ}$ 


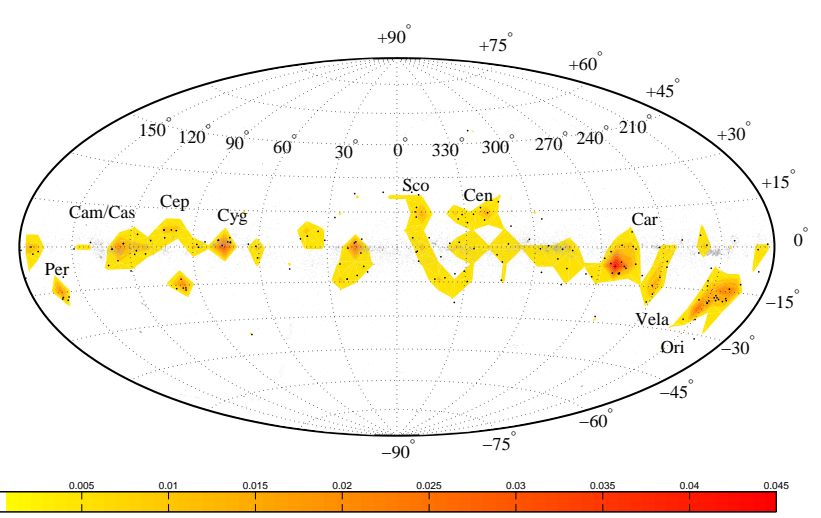

Fig. 11 The spatial distribution of regions with a local increased SN rate within $600 \mathrm{pc}$ from the sun, for the next $10 \mathrm{Myr}$; all SN events (black dots) will occur in $8.6 \%$ of the whole sky and $90 \%$ of the SN progenitors are distributed among $7.2 \%$ of the whole sky. The bin size is $7^{\circ} \times 7^{\circ}$.

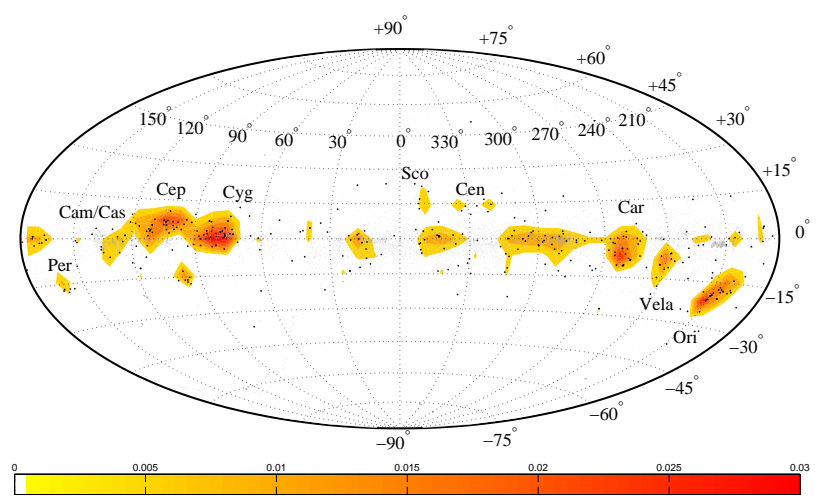

Fig. 12 The spatial distribution of regions with a local increased SN rate within $1 \mathrm{kpc}$ for the next $10 \mathrm{Myr}$; all SN progenitors (black dots) are distributed among $12.2 \%$ area of the whole sky and $90 \%$ of the SN events are in $9.5 \%$ of the whole sky. The bin size is $7^{\circ} \times 7^{\circ}$.

in Figure 11. The SN rate is increased for OB clusters like Carina, Orion, Vela, Scorpius, Centaurus, Cygnus, Cepheus, and Perseus, which all belong to the Gould Belt. All SN progenitors are distributed among $8.6 \%$ area of the whole sky and $90 \%$ of the progenitors are within $7.2 \%$ area of the whole sky.

Within $1 \mathrm{kpc}$ (Figure 12) all SN events will occur in $12.2 \%$ of the whole sky and $90 \%$ of the SNe are in $9.5 \%$ of the whole sky.

The regions with a locally increased $\mathrm{SN}$ rate within $5 \mathrm{kpc}$ from the sun are plotted in Figure 13. All SN progenitors are distributed among $21.0 \%$ area of the whole sky and $90 \%$ of the SN events will occur in $12.2 \%$ area of the whole sky.

While our sample is incomplete outside $\approx 1 \mathrm{kpc}$, the missing SN progenitors are possibly mostly in the same areas as the known ones. These results can be used e.g. for reducing the parameter space of Einstein@home, because GW sources or young neutron stars should be concentrated

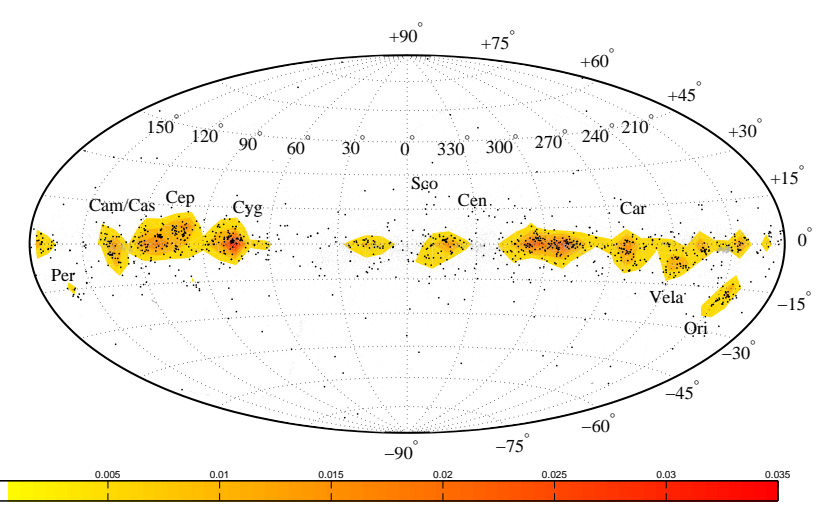

Fig. 13 The spatial distribution of regions with local increased SN rate within $5 \mathrm{kpc}$ for the next $10 \mathrm{Myr}$; all SN events (black dots) in $21.0 \%$ of the whole sky and $90 \% \mathrm{SNe}$ in $12.1 \%$ of the whole sky. The bin size is $7^{\circ} \times 7^{\circ}$.

in our marked regions, even when taking into account high kick velocities (Palomba 2005). Thus a blind search for these sources could be reduced to our regions.

The temporally resolved ccSN rate for the next $10 \mathrm{Myr}$ within $600 \mathrm{pc}$ yields $17.0_{-3.6}^{+4.5} \mathrm{ccSNe} / \mathrm{Myr}$ and is shown in Figure 14 Our result can be compared to the range from 20 to $27 \mathrm{ccSNe}$ per Myr for the Gould Belt from Grenier (2004) and also with $21 \pm 5 \mathrm{ccSNe} / \mathrm{Myr}$ from Hohle et al. (2010). The value between 2 and $3 \mathrm{Myr}$, seen as a peak in Hohle et al. (2010), is now consistent with a constant rate. Within 2 Myr the SN rate seems to be slightly below the average.

The true $\mathrm{SN}$ rate can be larger due to $\mathrm{SNe}$ of type Ia, $(\approx 15-25 \%$ of all $\mathrm{SNe}$ ) plus more unknown multiple massive companions. For these stars we take into account the probability of putative $8 M_{\odot}$ companions with the same age as the host star by using the binary fraction from Oudmaijer \& Parr (2010). This slight increase is illustrated by the grey dots in Figure 14

Many neutron stars are expected to be nearby the Galactic Center, but there is no major contribution to the spatial distribution in that direction due to high uncertainties in the parallax and high extinction values. The accurate photometric and astrometric results of the Gaia mission (de Bruijne 2012) could improve the completeness and the precision of our work significantly.

We consider stars with a mass higher than $8 M_{\odot}$ as SN progenitors and with a mass higher than $30 M_{\odot}$ as probable black hole (BH) progenitors (Woosley et al. 2002). In Table 8 we give the derived ccSN rate, the numbers of SN progenitors, and $\mathrm{BH}$ progenitors within a certain distance. We calculate the Galactic ccSN rate by extrapolating the local ccSN rate of our observed volume to the whole Galaxy as described in Reed (2005) using his Equations (5) and (16). We use as distance to the Galactic Center $8.5 \mathrm{kpc}$, a thick disc scale height of $0.9 \mathrm{kpc}$, and a scale length of $3.6 \mathrm{kpc}$ (Jurić et al.2008). The observed volume is then cal- 


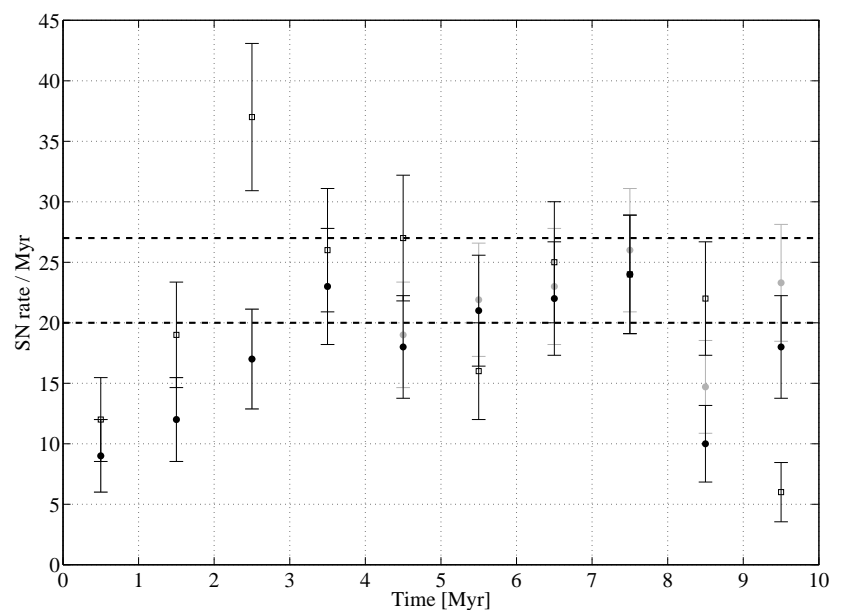

Fig. 14 The ccSN rate of $17.0_{-3.6}^{+4.5} \mathrm{ccSNe} / \mathrm{Myr}$ from this work for the next $10 \mathrm{Myr}$ within $600 \mathrm{pc}$ (black filled dots with Poissonian errorbars) in comparison with the results in Hohle et al. (2010) (black open squares). The dashed line indicates the apparent range for the Gould Belt from Grenier (2004). The increase of the SN rate due to putative massive companions of possible multiple stars is shown by grey dots.

culated within the disc using the distances given in Table 8 Comparing the ccSN rate in the Solar vicinity with the Galactic ccSN rate of 2.5 SNe per century (Tammann et al. 1994) suggests that the current ccSN rate within $600 \mathrm{pc}$ and $1 \mathrm{kpc}$ is increased due to the Gould Belt as found by Grenier (2004) and Popov et al. (2005) by about a factor of 5-6.

\section{Conclusions}

The sample contains one order of magnitude more stars than Hohle et al. (2010), is more complete, and includes more recent input parameters. Our consistency checks for the mass luminosity relation, the mass function, and the completeness confirmed known relations and published values from different authors. We found regions with a locally increased ccSN rate implying a higher probability of the presence of young neutron stars. The blind search for young nearby neutron stars and GW sources can work more efficient, if the parameter space of an all sky survey can be reduced to our predicted areas. Given the results of this work a blind search for GW sources and young neutron stars within 600 pc could be decreased to only $9 \%$ area of the whole sky. Regarding our highly incomplete sample within $5 \mathrm{kpc}$, the all sky survey could be restricted to $21 \%$ area of the whole sky. The discovery of new radio quiet neutron stars, like in Pires et al. (2009) in Carina, which shows an enhanced probability in our maps, is more probable in these special regions than in a blind search.

\section{Acknowledgements}

The authors would like to thank S. B. Popov, R. Chini, and V. V. Hambaryan for useful discussions, the Deutsche Forschungsgemeinschaft (DFG) for support in the Collaborative Research Center Sonderforschungsbereich Transregio 7 "Gravitationswellenastronomie", Bruce Allen \& Bernard Schutz for motivating and suggesting this study, and Nina Tetzlaff for helping with plotting issues.

This research has made use of the SIMBAD database; data products from the Two Micron All Sky Survey, which is a joint project of the University of Massachusetts and the Infrared Processing and Analysis Center/California Institute of Technology, funded by the National Aeronautics and Space Administration and the National Science Foundation; the Washington Double Star Catalog maintained at the U.S. Naval Observatory.

\section{References}

Aasi, J., Abadie, J., Abbott, B. P., et al.: 2013, PhRvD, 87, 042001

Ackermann, M., Ajello, M., Allafort, A., et al.: 2013, Science, 339, 807

Andersen, J.: 1991, A\&ARv 3, 91

Bertelli, G., Bressan, A., Chiosi, C., et al.: 1994, A\&AS 106,275

Bessell, M. S., Castelli, F., Plez, B.: 1998, A\&A 333, 231

Bessell, M. S., Brett, J. M.: 1988, PASP 100, 1134

Bondarenko, I. I., Perevozkina, E. L.: 1996, Bondarenko, I. I., \& Perevozkina, E. L.: 1996, Odessa Astronomical Publications 9, 20

Brancewicz, H. K., Dworak, T. Z.: 1980, ACTAA 30, 501

Cardelli, J. A., Clayton, G. C., Mathis, J. S.: 1989, ApJ 345, 245

Claret, A.: 2004, A\&A 424, 919

de Bruijne, J. H. J.: 2012, ApSS 341, 31

Diehl, R., Halloin, H., Kretschmer, K., et al.: 2006, Nat. 439, 45

Docobo, J. A., Andrade, M.: 2006, ApJ 652, 681

Docobo, J. A., \& Andrade, M.: 2013, MNRAS 428, 321

Dommanget, J., Nys, O.: 2002, CCDM (Catalog of Components of Double \& Multiple stars), VizieR Online Data Catalog 1274

Gould, B. G.: 1879, Resultados del Observatorio Nacional Argentino, 1, 14

Grenier, I. A.: 2004, arXiv, astro-ph/0409096

Harding, P., Morrison, H. L., Olszewski, E. W., et al.: 2001: AJ, 122, 1397

Hilditch, R. W.: 2001, An Introduction to Close Binary Stars Hoeg, E., Bässgen, G., Bastian, U., et al.: 1997, A\&A 323, L49

Hohle, M. M., Neuhäuser, R., Schutz, B.F.: 2010, AN 331, 349

Jurić, M., Ivezić, Ž., Brooks, A., et al.: 2008, ApJ 673, 864 
Table 8 The number of SN progenitors with $M>8 M_{\odot}$ and BH progenitors with $M>30 M_{\odot}$ within a specific distance. The lower number is obtained by subtracting the $1 \sigma$ error from the masses and the upper value is obtained by adding the $1 \sigma$ error to the masses. The ccSN rate is given for those progenitors which should explode within the next $10 \mathrm{Myr}$, the errors are $3 \sigma$. In this table we give the calculated ccSN rate for our Galaxy by extrapolating from the local SN rate (given in that row) to the whole Galaxy, with a distance to the Galactic Center of $8.5 \mathrm{kpc}$, a thick disc scale height of $0.9 \mathrm{kpc}$, and a scale length of $3.6 \mathrm{kpc}$ (Jurić et al. 2008). In the last column we give the completeness from Figure 10

\begin{tabular}{llllll}
\hline $\begin{array}{l}\text { distance } \\
{[\mathrm{kpc}]}\end{array}$ & $\begin{array}{l}\text { SN } \\
{[\# \text { progenitors }]}\end{array}$ & $\begin{array}{l}\text { BH } \\
{[\# \text { progenitors }]}\end{array}$ & $\begin{array}{l}\text { SN rate } \\
{[\text { SN/Myr }]}\end{array}$ & $\begin{array}{l}\text { Gal.SN rate } \\
{[\text { SN/100yr }]}\end{array}$ & $\begin{array}{l}\text { Completeness } \\
{[\%]}\end{array}$ \\
\hline$\leq 0.6$ & $273_{-28}^{+27}$ & $0 \pm 0$ & $16.9_{-3.6}^{+4.5}$ & $11.9_{-0.9}^{+1.9}$ & 85 \\
\hline$\leq 1.0$ & $518_{-47}^{+44}$ & $2 \pm 0$ & $34.8_{-6.9}^{+6.0}$ & $6.0_{-0.4}^{+0.3}$ & 45 \\
\hline$\leq 3.0$ & $763_{-63}^{+62}$ & $6 \pm 1$ & $57.6_{-9.6}^{+7.8}$ & $0.7_{-0.1}^{+0.1}$ & 6 \\
\hline$\leq 5.0$ & $1328_{-111}^{+78}$ & $58_{-7}^{+4}$ & $110.2_{-15.6}^{+9.0}$ & $0.4_{-0.1}^{+0.1}$ & 2 \\
\hline
\end{tabular}

Kenyon, S. J., Hartmann, L.: 1995, ApJS 101, 117

Kharchenko, N. V., Roeser, S.: 2009, All-sky Compiled Catalogue of 2.5 million stars, VizieR Online Data Catalog 1280

Kodama, T.: 1997, PhD thesis, Institute of Astronomy, Univ. Tokyo

Kroupa, P., Tout, C. A., Gilmore, G.: 1993, MNRAS 262, 545

Lang, K. R.: 1992, Astrophysical Data I. Planets and Stars.

Lynga, G.: 1982, A\&A 109, 213

Maeder, A., Meynet, G.: 1989, A\&A 210, 155

Manchester, R. N., Hobbs, G. B., Teoh, A., \& Hobbs, M.: 2005, AJ 129, 1993

Mason, B. D., Wycoff, G. L., Hartkopf, W. I., et al.: 2010, The Washington Visual Double Star Catalog, VizieR Online Data Catalog 1020

Meynet, G. and Maeder, A.: 2003, A\&A 404, 975

Mihalas, D., \& Binney, J.: 1981: San Francisco, CA, W. H. Freeman and Co., 1981. 608 p.,

Ochsenbein, F., Bauer, P., Marcout, J.: 2000, A\&AS 143, 23

Oudmaijer, R. D., \& Parr, A. M.: 2010, MNRAS 405, 2439

Palomba, C.: 2005, MNRAS 359, 1150

Perevozkina, E. L., Svechnikov, M. A.: 1999, Catalog of eclipsing binaries parameters, VizieR Online Data Cata$\log 5118$

Perryman, M. A. C., Lindegren, L., Kovalevsky, J., et al.: 1997, A\&A 323, L49

Pires, A. M. and Motch, C. and Turolla, R. and Treves, A. and Popov, S. B.,: 2009 A\&A 498, 233

Popov, S. B., Turolla, R., Prokhorov, M. E., Colpi, M., \& Treves, A.: 2005, ApSS 299, 117

Pourbaix, D., Tokovinin, A. A., Batten, A. H., et al.: 2004, A\&A 424, 727

Reed, B. C.: 2005, AJ, 130, 1652

Rieke, G. H., Lebofsky, M. J.: 1985, ApJ 288, 618

Savage, B. D., Mathis, J. S.: 1979, ARAA 17, 73

Schaller, G., Schaerer, D., Meynet, G., et al.: 1992, A\&AS 96, 269

Schröder, S. E., Kaper, L., Lamers, H. J. G. L. M., et al.: 2004, A\&A 428, 149

Smith, Jr. H., Eichhorn, H.: 1996, MNRAS 281, 211
Skiff, B. A.: 2013, Catalogue of Stellar Spectral Classifications, VizieR Online Data Catalog 2023

Skrutskie, M. F., Cutri, R. M., Stiening, R., et al.: 2006, AJ 131, 1163

Stothers, R., \& Frogel, J. A.: 1974, AJ 79, 456

Sota, A., Maíz Apellániz, J., Walborn, N. R. et al.: 2011, ApJS 193,24

Surkova, L. P., Svechnikov, M. A.: 2004, Semi-detached eclipsing binaries, VizieR Online Data Catalog 5115

Svensmark, J., Enghoff, M. B., Svensmark, H.: 2012, ACPD 12,3595

Tammann, G. A., Loeffler, W., Schroeder, A.: 1994, ApJS 92,487

Torres, G.: 2010, AJ 140, 1158

van der Hucht, K. A.: 2001, NewAR 45, 135

van Leeuwen, F.: 2007, A\&A 474, 653

Wenger, M., Ochsenbein, F., Egret, et al.: 2000, A\&AS 143, 9

Wegner, W.: 2007, MNRAS 374, 1549

Woosley, S. E., Heger, A., \& Weaver, T. A.: 2002, Reviews of Modern Physics, 74, 1015

Zacharias, N., Monet, D. G., Levine, S. E., et al.: 2005, NOMAD Catalog, VizieR Online Data Catalog 1297

Zacharias, N., Finch, C. T., Girard, T. M., et al.: 2013, AJ 145,44

Zinnecker, H. and Yorke, H. W.: 2007, ARAA 45, 481

\section{A Selection of the stars}

The basic set of stars comes from a select by criteria query in SIMBAD using the following search expression:

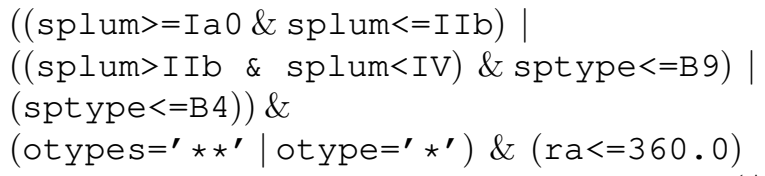

where splum defines the spectral LC, sptype specifies the spectral type, otype is for the classification of the objects e.g. as stars or multiple stars, and the ra selection is to query stars with coordinates. The last argument 


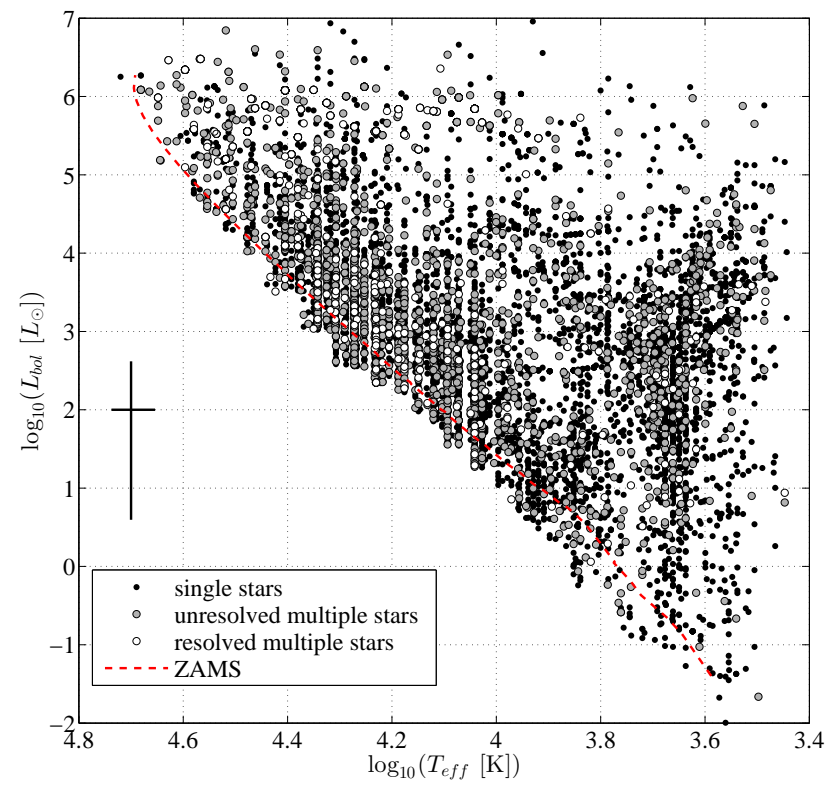

Fig. B1 Our derived H-R D: the red dashed line marks the zero age main sequence (ZAMS), grey dots indicate resolved stars in a multiple system, black circles are unresolved multiples, and black dots identify single stars, the mean errorbars are indicated by the black lines on the left side.

in Equation A1 filters out 410 stars without listed coordinates. We expand the sample from SIMBAD by querying catalogues containing spectral classifications in the order of descending priority: Sota et al. (2011), CSSC by Skiff (2013), ASCC-2.5 by Kharchenko \& Roeser (2009), HIPPARCOS by Perryman et al. (1997). The VizieR Online Database (Ochsenbein et al. 2000) was used to download these catalogues.

\section{B Calculations and Results without transformed Parallax}

In this section we give all results derived without applying Eq. (21) from Smith \& Eichhorn (1996). The values are consistent within their errorbars compared to the values in the main part of the paper.

The calculated H-RD using spectral classification and Equation 3 is seen in Figure B1. There are in total 4504 stars below the zero-age-main-sequence (ZAMS) due to large uncertainties of the distance. Those stars are flagged and assumed to have at least a luminosity consistent with the ZAMS. From our 6407 stars with sufficiently measured distances subsection 3.4, 518 stars are below the ZAMS.

The error-weighted fit for the mass luminosity relation of main sequence stars (Figure B2) yields $\beta=4.14 \pm$ 0.92 and is consistent with $\beta=4.0$ for stars with $M \leq$ $10 M_{\odot}$ and $\beta=3.6$ for stars with a higher mass from Hilditch (2001), $\beta=3.84$ from Andersen (1991), and with $\beta=3.66 \pm 0.12$ from Hohle et al. (2010) The mass func-

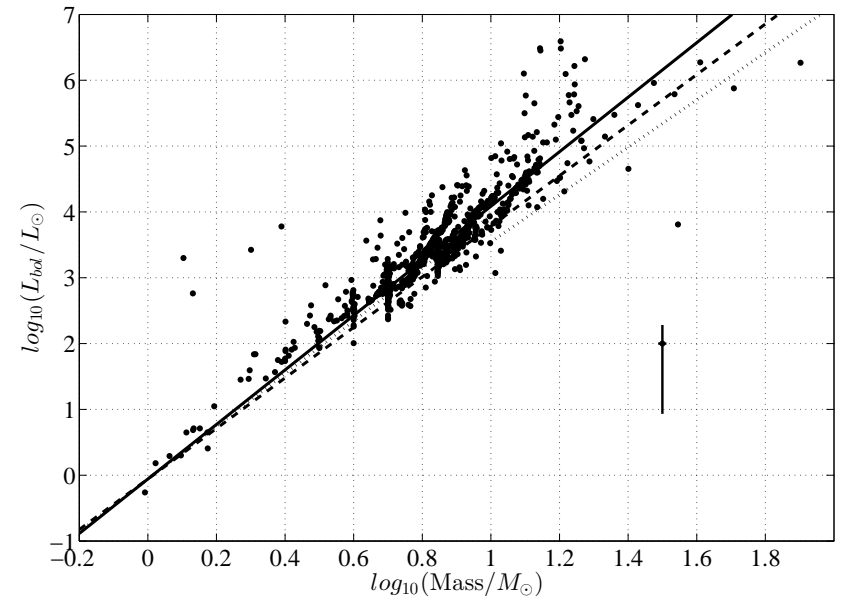

Fig. B2 Derived error weighted mass luminosity relation $L \sim M^{\beta}$ with $\beta=4.14 \pm 0.92$ (solid line) for main sequence stars compared to with $\beta=4.0$ for stars with $M \leq$ $10 M_{\odot}$ and 3.6 for stars with a higher mass (dotted line) from Hilditch (2001), and $\beta=3.84$ from Andersen (1991) (dashed line). Our result is consistent with $\beta=3.66 \pm 0.12$ from Hohle et al. (2010). The black line indicates the mean errorbar.

Table B1 List of $L_{b o l}$, mass, age, remaining life-time $\tau_{r}$, and number $\mathrm{N}$ of known additional components of the first ten SN progenitors in Table 3, with $1 \sigma$ errors. Uncertain remaining life-times are marked with a leading colon.

\begin{tabular}{llrrrrr}
\hline$\#$ & name & $\begin{array}{r}L_{\text {bol }} \\
10^{3}\left[L_{\odot}\right]\end{array}$ & $\begin{array}{r}\text { mass } \\
{\left[M_{\odot}\right]}\end{array}$ & $\begin{array}{r}\text { age } \\
{[\mathrm{Myr}]}\end{array}$ & $\begin{array}{r}\tau_{r} \\
{[\mathrm{Myr}]}\end{array}$ & $\mathrm{N}$ \\
\hline 1 & $\alpha$ Mus & $5.4 \pm 2.4$ & $8.6(2)$ & $16 \pm 3$ & 13 & 4 \\
2 & $\eta$ Cen & $7.4 \pm 3.5$ & $8.8(2)$ & $20 \pm 3$ & 8 & 0 \\
3 & $\beta$ Lup & $6.8 \pm 3.2$ & $8.3(3)$ & $28 \pm 5$ & $: 11$ & 1 \\
4 & HR 4898 & $3.8 \pm 1.7$ & $8.9(1)$ & $3 \pm 1$ & 24 & 1 \\
5 & $\zeta$ Oph & $22.4 \pm 2.5$ & $12.5(1)$ & $14 \pm 1$ & 2 & 0 \\
6 & $\alpha$ Lup & $15.7 \pm 7.5$ & $9.6(2)$ & $23 \pm 1$ & $: 9$ & 2 \\
7 & HR 5193 & $6.9 \pm 3.3$ & $8.7(2)$ & $19 \pm 4$ & 9 & 2 \\
8 & $\phi$ Cen & $4.7 \pm 2.3$ & $8.4(2)$ & $14 \pm 2$ & 16 & 0 \\
9 & $\zeta$ Cas & $6.3 \pm 3.1$ & $8.2(2)$ & $28 \pm 5$ & 7 & 0 \\
10 & $v$ Sco & $16.4 \pm 8.1$ & $9.7(3)$ & $23 \pm 2$ & $: 4$ & 0 \\
\hline
\end{tabular}

tion Figure B3 yields $\Gamma=-2.8 \pm 0.7$ for stars with $M \gtrsim 8 M_{\odot}$ and is consistent with $\Gamma=-2.7 \pm 0.7$ from Kroupa et al. (1993). The calculation of mass, age and remaining life-time, like in section 4 , produces 6705 stars with a negative remaining life-time. From the subsample of stars with sufficient distances subsection 3.4) 2450 are corrected to positive remaining life-times. 


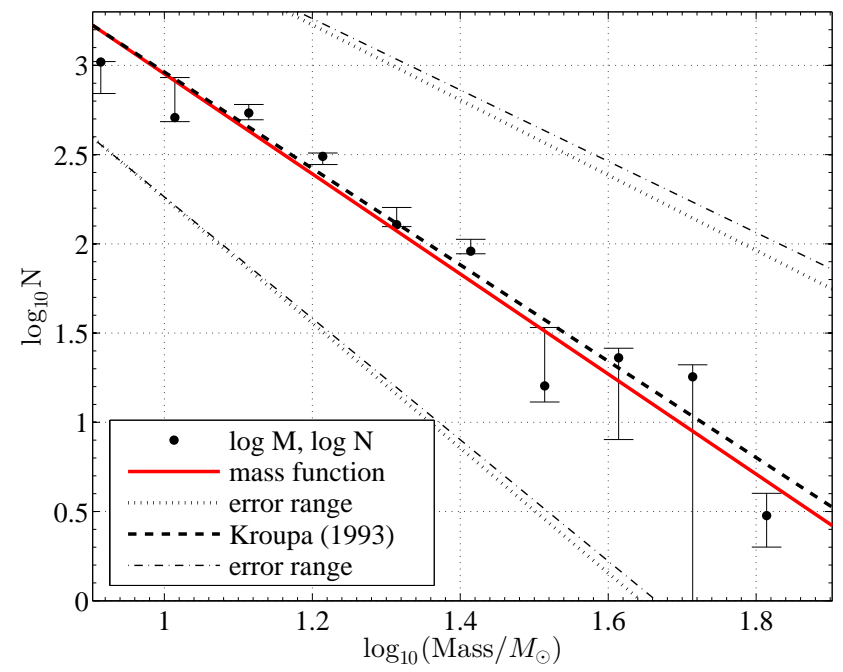

Fig. B3 Our derived mass function $N \sim M^{\Gamma}$ with $\Gamma=$ $-2.8 \pm 0.7$ for stars with $M \gtrsim 8 M_{\odot}$ (red line), wihout applied transformation by Smith \& Eichhorn (1996), compared to the initial mass function from Kroupa et al. (1993) with $\Gamma=-2.7 \pm 0.7$ (dashed black line). The errorbars of the individual masses (black dots) indicate the $1 \sigma$ confidence interval.

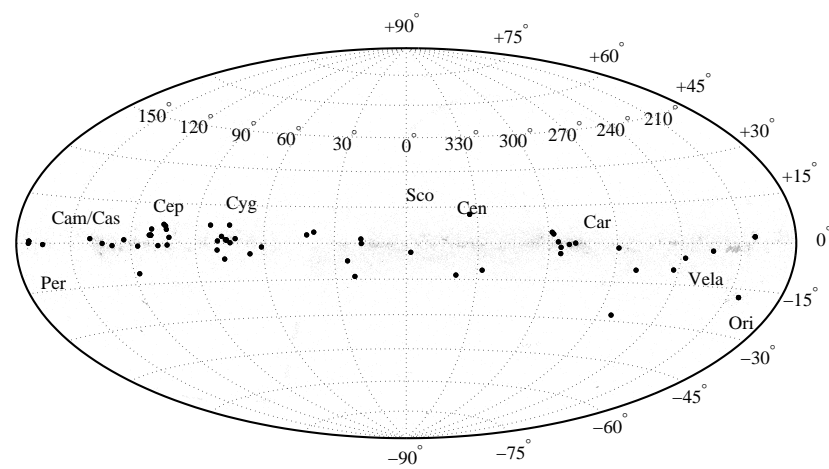

Fig. B4 The spatial distribution of 130 binaries in galactic coordinates, where both components have at least $8 M_{\odot}$ (black dots) compared to the entire sample (grey dots).

The spatial distribution of 130 binaries, where both components have $M>8 M_{\odot}$ is plotted in Figure B4. The regions with a locally increased $\mathrm{SN}$ rate within a range of up to $600 \mathrm{pc}$ are seen in Figure B5 All SN events will occur in $8.3 \%$ area of the whole sky and $90 \%$ SN events in $7.1 \%$ area of the whole sky. The distribution within $1 \mathrm{kpc}$ yields that all SN progenitors are in $12.0 \%$ of the whole sky and $90 \%$ SN progenitors are in $9.5 \%$ of the whole sky (Figure B6). Within $5 \mathrm{kpc}$ (Figure B7) all SN progenitors are distributed among $20.3 \%$ area of the whole sky and $90 \% \mathrm{SNe}$ are in $12.1 \%$ area of the whole sky. The temporally resolved $\mathrm{SN}$ rate of $19.1_{-5.4}^{+4.2}$ (Figure B8) can be compared to the apparent lower limit of $20 . .27 \mathrm{ccSNe} / \mathrm{Myr}$ for the Gould Belt from Grenier (2004) and to $\approx 20 \mathrm{ccSNe} / \mathrm{Myr}$ from Hohle et al. (2010).

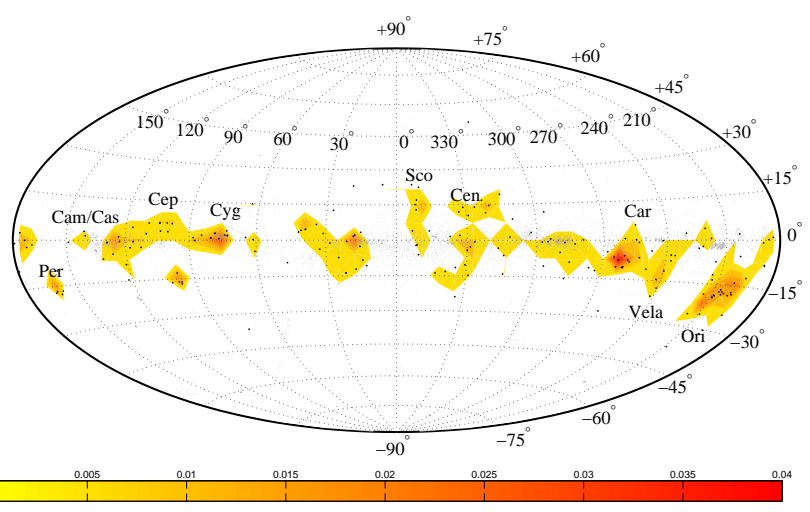

Fig. B5 The spatial distribution of areas with a local increased SN rate within $600 \mathrm{pc}$; all SN events will occur in $8.3 \%$ area of the whole sky and $90 \%$ SNe in $7.1 \%$ area of the whole sky. The bin size is $7^{\circ} \times 7^{\circ}$.

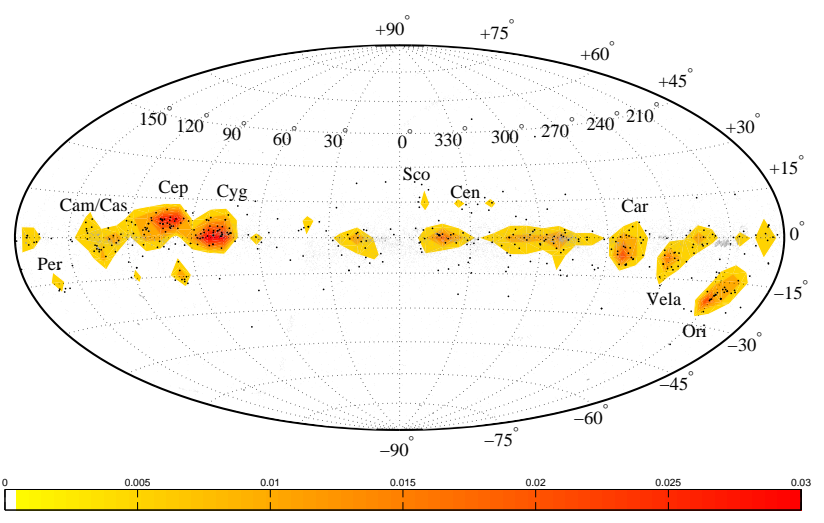

Fig. B6 The spatial distribution of areas with local increased SN rate within $1 \mathrm{kpc}$; all $\mathrm{SN}$ events will occur in $12.0 \%$ area of the whole sky and $90 \% \mathrm{SNe}$ in $9.5 \%$ area of the whole sky. The bin size is $7^{\circ} \times 7^{\circ}$.

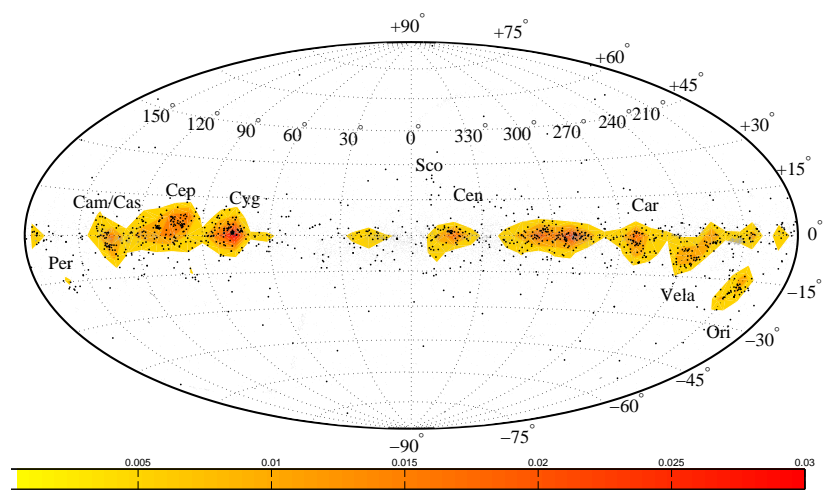

Fig. B7 The spatial distribution of areas with local increased SN rate within $5 \mathrm{kpc}$; all SN events in $20.3 \%$ area of the whole sky and $90 \% \mathrm{SNe}$ in $12.1 \%$ area of the whole sky. The bin size is $7^{\circ} \times 7^{\circ}$. 
Table B2 The number of ccSN progenitors with $M>8 M_{\odot}$ and BH progenitors with $M>30 M_{\odot}$ within a specific distance. The lower number is obtained by subtracting the $1 \sigma$ error from the masses and the upper value is obtained by adding the $1 \sigma$ error to the masses. The ccSN rate is given for those progenitors which should explode within the next $10 \mathrm{Myr}$, the errors are $3 \sigma$. In this table we give the calculated ccSN rate for our Galaxy by extrapolating from the local ccSN rate (given in that row) to the whole Galaxy, with a distance to the Galactic center of $8.5 \mathrm{kpc}$, a thick disc scale height of $0.9 \mathrm{kpc}$, and a scale length of $3.6 \mathrm{kpc}$ (Jurić et al. 2008). In the last column we give the completeness from Figure 10.

\begin{tabular}{llllll}
\hline $\begin{array}{l}\text { distance } \\
{[\mathrm{kpc}]}\end{array}$ & $\begin{array}{l}\text { SN } \\
{[\# \text { progenitors }]}\end{array}$ & $\begin{array}{l}\text { BH } \\
{[\# \text { progenitors }]}\end{array}$ & $\begin{array}{l}\text { SN rate } \\
{[\mathrm{SN} / \mathrm{Myr}]}\end{array}$ & $\begin{array}{l}\text { Gal.SN rate } \\
{[\mathrm{SN} /(100 \mathrm{yr})]}\end{array}$ & $\begin{array}{l}\text { Completeness } \\
{[\%]}\end{array}$ \\
\hline$\leq 0.6$ & $286_{-35}^{+35}$ & $0_{-0}^{+0}$ & $19.1_{-5.4}^{+4.2}$ & $13.4_{-3.8}^{+3.0}$ & 85 \\
\hline$\leq 1.0$ & $574_{-58}^{+51}$ & $2_{-0}^{+1}$ & $42.3_{-9.3}^{+7.2}$ & $7.3_{-1.6}^{+1.2}$ & 45 \\
\hline$\leq 3.0$ & $816_{-93}^{+84}$ & $13_{-2}^{+4}$ & $65.6_{-17.7}^{+9.3}$ & $0.8_{-0.2}^{+0.1}$ & 6 \\
\hline$\leq 5.0$ & $1294_{-134}^{+101}$ & $56_{-9}^{+7}$ & $109.9_{-24.9}^{+10.5}$ & $0.4_{-0.1}^{+0.1}$ & 2 \\
\hline
\end{tabular}

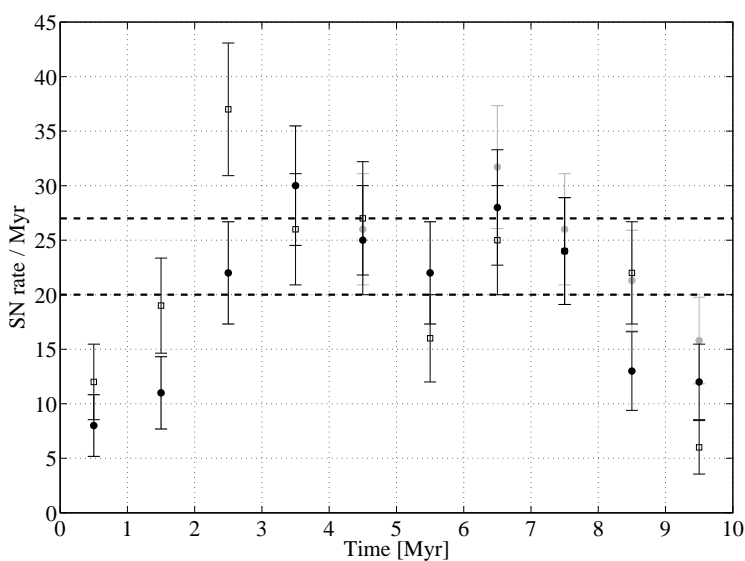

Fig. B8 The ccSN rate of $19.1_{-5.4}^{+4.2} \mathrm{ccSNe} / \mathrm{Myr}$ (Poissonian errorbars) from this work for the next $10 \mathrm{Myr}$ within $600 \mathrm{pc}$ (black dots) in comparison with the results in Hohle et al. (2010) (black squares). The dashed line indicates the apparent lower limits for the Gould Belt from Grenier (2004). The increase of the ccSN rate due to putative massive companions of possible multiple stars is shown by grey dots.

\section{Massive binary systems}

Table C2 List of those $6 \mathrm{SN}$ progenitors within $3 \mathrm{kpc}$ which might be also BH progenitors (see Table 8): SIMBAD identifier, distance, and spectral type from Table 3 , mass and remaining life-time $\tau_{r}$ from Table 7

\begin{tabular}{lllll}
\hline name & spec.type & $\begin{array}{l}\text { distance } \\
{[\mathrm{kpc}]}\end{array}$ & $\begin{array}{l}\text { mass } \\
{\left[M_{\odot}\right]}\end{array}$ & $\begin{array}{l}\boldsymbol{\tau}_{\boldsymbol{r}} \\
{[\mathrm{Myr}]}\end{array}$ \\
\hline V973 Sco & O8Iaf & $1.7_{-0.5}^{+1.1}$ & $35.0 \pm 1.7$ & 2.5 \\
V453 Sco & B1Ia(e)p & $1.4_{-0.5}^{+1.2}$ & $31.0 \pm 0.3$ & 2.3 \\
9 Sgr & O4V & $1.6_{-0.5}^{+1.3}$ & $73 \pm 14$ & 2.8 \\
HD 14434 & O5.5V & $2.8_{-1.4}^{+13}$ & $33.5 \pm 8.5$ & 4.0 \\
Naos & O6/7III & $0.33 \pm 0.01$ & $35.6 \pm 3.8$ & 2.0 \\
VV Cep & M3:Ia-Iab: & $0.7 \pm 0.1$ & $63.8 \pm 0.6$ & 2.4 \\
\hline
\end{tabular}


Table C1 List of those 35 Binaries $^{a}$ with at least two massive components, which were also studied in Hohle et al. (2010), with updated parameters: SIMBAD identifier and distance from Table 3, spectral types and dynamical mass from [1] Bondarenko \& Perevozkina (1996), [2] Brancewicz \& Dworak (1980), [3] Docobo \& Andrade (2006), [4] Perevozkina \& Svechnikov (1999), [5] Pourbaix et al. (2004), [6] Surkova \& Svechnikov (2004), [7] Dommanget \& Nys (2002), and [8] Mason et al. (2010).

\begin{tabular}{|c|c|c|c|c|c|c|c|}
\hline \multirow[t]{2}{*}{$\#$} & \multirow[t]{2}{*}{ name } & \multirow{2}{*}{$\begin{array}{c}\text { distance } \\
{[\mathrm{pc}]}\end{array}$} & \multicolumn{2}{|c|}{ spectral type } & \multicolumn{2}{|c|}{$\operatorname{mass}\left[M_{\odot}\right]$} & \multirow[t]{2}{*}{ ref. } \\
\hline & & & primary & secondary & prim. & sec. & \\
\hline 1 & $\mathrm{AO}$ Cas & $717_{-167}^{+313}$ & O7-O9III-O9III & O8/9-O9III-O9III & $20.30-31.73$ & $14.80-26.02$ & {$[1][2][5]$} \\
\hline 2 & BM Cas & $515_{-150}^{+360}$ & A5I-A5Ia & G0I & 19.88 & 9.95 & [2][5] \\
\hline 3 & CC Cas & $553_{-137}^{+271}$ & O9.3IV-O9IV & O9IV-O9IV & 20.37 & 9.98 & [2][5] \\
\hline 4 & IU Aur & $216_{-47}^{+83}$ & $\begin{array}{l}\mathrm{O} 9.5 \mathrm{~V}-\mathrm{B} 0: \mathrm{PV}- \\
\mathrm{B} 0-\mathrm{B} 3-\mathrm{B} 3 \mathrm{Vnne}\end{array}$ & $\mathrm{B} 0.5-\mathrm{B} 2-\mathrm{B} 1$ & $12.04-21.30$ & $7.94-14.50$ & {$[1][2][5][7][8]$} \\
\hline 5 & LY Aur & $252_{-77}^{+197}$ & $\begin{array}{l}\mathrm{O} 9.5 \mathrm{III}-\mathrm{O} 9.5- \\
\mathrm{O} 9.5 \mathrm{III}-\mathrm{B} 5- \\
\text { O9.5III }\end{array}$ & B0IV-O9.5III & $21.28-24.00$ & $12.70-18.17$ & {$[1][2][5][7][8]$} \\
\hline 6 & $\delta$ Pic & $394_{-22}^{+25}$ & $\begin{array}{l}\text { B3III-B0.5IV- } \\
\text { B0.5III }\end{array}$ & B3.5-B & $16.27-16.90$ & $8.63-9.00$ & {$[1][2][5]$} \\
\hline 7 & V641 Mon & $586_{-176}^{+438}$ & B1.5IV & B3 & 11.50 & 8.40 & [1] \\
\hline 8 & FZ CMa & $391_{-107}^{+238}$ & $\begin{array}{l}\text { B2.5IV-VN-B2.5IV- } \\
\text { V }\end{array}$ & B2.5IV-V & 15.35 & 15.36 & {$[2][5]$} \\
\hline 9 & FF CMa & $827_{-254}^{+662}$ & B3 & B4 & 11.10 & 8.88 & [2] \\
\hline 10 & UW CMa & $579_{-79}^{+108}$ & $\mathrm{O} 7-\mathrm{O} 7 \mathrm{f}-\mathrm{O} 7 \mathrm{f}$ & $\mathrm{O} 7.5-\mathrm{O} 9 \mathrm{III}-\mathrm{O} 7$ & $22.00-32.62$ & $18.30-24.47$ & {$[1][2][5]$} \\
\hline 11 & TU Mus & $1675_{-671}^{+3} 367$ & $\mathrm{O} 7-\mathrm{B} 3-\mathrm{O} 7-\mathrm{O} 8$ & $\mathrm{O} 9.5-\mathrm{B} 3$ & $22.39-22.60$ & $15.00-15.40$ & {$[1][2][5]$} \\
\hline 12 & VZ Cen & $1555_{-591}^{+2} 463$ & B1III: & $?$ & 14.58 & 10.21 & [2] \\
\hline 13 & W Cru & $1922_{-857}^{+79122}$ & G2IAB-G1Iab & $?$ & 11.67 & 8.18 & [2][5] \\
\hline 14 & V701 Sco & $\begin{array}{l}645_{-199}^{+517} \\
\end{array}$ & $\mathrm{~B} 1 \mathrm{~V}-\mathrm{B} 5-\mathrm{B} 2 \mathrm{nn}$ & B1.5 & $6.42-10.30$ & $5.53-10.2$ & {$[1][2][5]$} \\
\hline 15 & HD168206 & $774_{-250}^{+710}$ & WC7-WC8 & B0-O8-9III-V & 18.49 & 11.22 & {$[2][5]$} \\
\hline 16 & RZ Oph & $437_{-134}^{+347}$ & F3eIb-F5II-III & K5II-K5III & 18.46 & 11.14 & [2][5] \\
\hline 17 & V1182 Aql & $358_{-85}^{+164}$ & $\mathrm{O} 9 \mathrm{~V}-\mathrm{O} 9-\mathrm{O} 9 \mathrm{Vnn}$ & $\mathrm{B} 1-\mathrm{B} 3-\mathrm{B} 3 \mathrm{~V}$ & $18.01-38.20$ & $10.85-13.80$ & {$[1][2][5]$} \\
\hline 18 & V599 Aql & $214_{-23}^{+30}$ & $\mathrm{~B} 3.5-\mathrm{B} 4 \mathrm{~V}-\mathrm{B} 2 \mathrm{~V}$ & $\mathrm{~B} 3.5-\mathrm{B} 8$ & $18.03-18.40$ & $11.35-11.40$ & {$[1][2][5]$} \\
\hline 19 & $v \mathrm{Sgr}$ & $541_{-60}^{+78}$ & B8pI:-B8p & $\mathrm{F} 2 \mathrm{pI}-\mathrm{F} 2 \mathrm{pe}$ & 21.26 & 18.10 & [2][5] \\
\hline 20 & V380 Cyg & $643_{-87}^{+118}$ & B1III-B1.5II-III & $\mathrm{B} 3 \mathrm{~V}-\mathrm{B} 2 \mathrm{~V}$ & 16.70 & 9.36 & [2][5] \\
\hline 21 & V448 Cyg & $650_{-200}^{+519}$ & $\begin{array}{l}09.5 \mathrm{e}-08.9 \mathrm{~V}- \\
09.5 \mathrm{~V}\end{array}$ & $\begin{array}{l}\text { B1IB- } \\
\text { II-B1.2Ib-B1Ib }\end{array}$ & $23.84-25.20$ & $14.00-15.85$ & {$[2][5][6]$} \\
\hline 22 & V382 Cyg & $674_{-207}^{+538}$ & $\begin{array}{l}\mathrm{O} 6.5 \mathrm{~V}-\mathrm{O} 7.5- \\
\mathrm{O} 6.5-\mathrm{B} 0-\mathrm{B} 0\end{array}$ & $\mathrm{O} 7.5-\mathrm{O} 9-07.5$ & $28.00-37.16$ & $19.60-32.69$ & {$[1][2][5][7][8]$} \\
\hline 23 & V470 Cyg & $589_{-164}^{+368}$ & $\mathrm{~B} 2-\mathrm{B} 2$ & $\mathrm{~B} 2.5-\mathrm{B} 2$ & 13.82 & 12.16 & {$[2][5]$} \\
\hline 24 & V444 Cyg & $604_{-163}^{+355}$ & O8III:-B1 & WN5.5-WN5 & 34.53 & 19.33 & [2][5] \\
\hline 25 & V729 Cyg & $499_{-153}^{+398}$ & $\mathrm{O} 7-\mathrm{O} 7 \mathrm{f}-\mathrm{O} 7 \mathrm{f}-\mathrm{O} 7 \mathrm{e}$ & $\mathrm{O} 9-\mathrm{B} 0-\mathrm{O} 8-\mathrm{O} 6 \mathrm{f}$ & $26.70-27.80$ & $6.70-23.00$ & {$[1][2][5][8]$} \\
\hline 26 & V367 Cyg & $567_{-110}^{+183}$ & $\begin{array}{l}\text { A5EpIA-A5Iab- } \\
\text { F5-A7Iape+F5 }\end{array}$ & $\mathrm{A} 9-\mathrm{A} 3$ & 12.62 & 8.84 & {$[2][5][7][8]$} \\
\hline 27 & Y Cyg & $1118_{-330}^{+804}$ & B0IV-BOIV & B0IV-B0IV & 17.71 & 17.54 & {$[2][5]$} \\
\hline 28 & V1488 Cyg & $321_{-34}^{+44}$ & $\begin{array}{l}\text { K5IA-K5Iab- } \\
\text { K0-K3Ib }\end{array}$ & $\mathrm{B} 4 \mathrm{~V}-\mathrm{B} 4 \mathrm{IV}-\mathrm{V}-\mathrm{B} 3 \mathrm{~V}$ & 22.64 & 8.16 & {$[2][5][7][8]$} \\
\hline 29 & HD208392 & $485_{-99}^{+169}$ & $\mathrm{~B} 0.5 \mathrm{~V}-\mathrm{B} 3-\mathrm{B} 1 \mathrm{~V}$ & B1VE & 10.51 & 9.46 & {$[2][7][8]$} \\
\hline 30 & VV Cep & $744_{-97}^{+132}$ & $\begin{array}{l}\text { M2pIAE- } \\
\text { M2epIa-M1- } \\
\text { M2Iape }\end{array}$ & B8VE-B9 & 63.81 & 35.09 & {$[2][5][7][8]$} \\
\hline 31 & HD 211853 & $428_{-109}^{+221}$ & B0:III:-O & WN6-WN6 & 23.95 & 16.12 & [2][5] \\
\hline 32 & DH Cep & $927_{-286}^{+749}$ & $\mathrm{O} 5-\mathrm{O} 6 \mathrm{n}-\mathrm{B} 2-\mathrm{O} 5$ & O5-O6n & 34.00 & 27.70 & {$[1][5][7][8]$} \\
\hline 33 & AH Cep & $981_{-252}^{+519}$ & $\mathrm{O} 8-\mathrm{B} 0.5 \mathrm{~V}-\mathrm{B} 0.5 \mathrm{Vn}$ & $\mathrm{O} 9-\mathrm{B} 0.5-\mathrm{B} 0.5 \mathrm{Vn}$ & $15.22-18.10$ & $13.23-15.90$ & {$[1][2][5]$} \\
\hline 34 & NY Cep & $1496_{-492}^{+1434}$ & $\begin{array}{l}\text { B0IV-BOIV- } \\
\text { B0-BOIV }\end{array}$ & BOIV-BOIV & 16.07 & 13.93 & {$[2][5][7][8]$} \\
\hline 35 & HD218066 & $602_{-164}^{+358}$ & $\begin{array}{l}\text { B } 0.5-\mathrm{B} 0.5 \mathrm{IV}- \\
\mathrm{V}-\mathrm{B} 1-\mathrm{B} 1: \mathrm{V}: \mathrm{var}\end{array}$ & B0.5IV-V-B0.5IV-V & 10.62 & 9.45 & {$[2][5][7][8]$} \\
\hline
\end{tabular}

\footnotetext{
${ }^{a}$ Since there are no coordinates given in Brancewicz \& Dworak (1980) a few HIPPARCOS stars were misidentified in Table 5 in Hohle et al. (2010). We use the correct coordinates in this work.
} 